\title{
Complementarity of LHC and EDMs for exploring Higgs CP violation
}

\author{
Chien-Yi Chen, ${ }^{a}$ S. Dawson ${ }^{a}$ and Yue Zhang $^{b}$ \\ ${ }^{a}$ Department of Physics, Brookhaven National Laboratory, \\ Upton, New York, 11973, U.S.A. \\ ${ }^{b}$ Walter Burke Institute for Theoretical Physics, California Institute of Technology, \\ Pasadena, CA 91125, U.S.A. \\ E-mail: cychen@bnl.gov, dawson@bnl.gov, yuezhang@caltech.edu
}

\begin{abstract}
We analyze the constraints on a CP-violating, flavor conserving, two Higgs doublet model from the measurements of Higgs properties and from the search for heavy Higgs bosons at LHC, and show that the stronger limits typically come from the heavy Higgs search channels. The limits on CP violation arising from the Higgs sector measurements are complementary to those from EDM measurements. Combining all current constraints from low energy to colliders, we set generic upper bounds on the $\mathrm{CP}$ violating angle which parametrizes the $\mathrm{CP}$ odd component in the $126 \mathrm{GeV}$ Higgs boson.
\end{abstract}

KeYwords: Higgs Physics, Beyond Standard Model, CP violation

ARXIV EPRINT: 1503.01114 


\section{Contents}

1 Introduction 1

2 Two Higgs doublet models and CP violation 3

2.1 Scalar potential with two Higgs doublets 3

2.2 Neutral scalar interactions 4

2.3 CP violation implies a non-decoupled heavy Higgs sector 4

2.4 Beyond approximate $Z_{2}$ symmetry 5

2.5 Production and decay of the heavy Higgs at LHC 6

2.5.1 Production 6

$\begin{array}{ll}2.5 .2 \text { Decays } & 7\end{array}$

$\begin{array}{lll}2.6 & \mathrm{CP} \text { violation and heavy Higgs signal rates } & 8\end{array}$

3 Results 10

3.1 Limits from heavy Higgs searches in approximate $Z_{2}$ symmetric models $\quad 12$

3.2 Limits from heavy Higgs searches in the models with no $Z_{2}$ symmetry 12

4 Limits from $B$ decays, oblique parameters, and $(g-2)_{\mu} \quad 20$

4.1 Limits from electroweak oblique parameters 20

$\begin{array}{lll}4.2 & \text { Limits from muon } g-2 & 22\end{array}$

5 Conclusion $\quad 24$

A Solving the potential parameters in the approximate $Z_{2}$ case 25

B Tri-linear Higgs couplings $\quad 25$

$\begin{array}{ll}\text { C Formula for } g-2 & 26\end{array}$

\section{Introduction}

Now that the $126 \mathrm{GeV}$ Higgs boson has been discovered [1, 2], the exploration of its properties is the focus of LHC phenomenology. The current measurements of Higgs production and decay rates are consistent with the Standard Model $(\mathrm{SM})$ predictions at the $\sim 10$ $20 \%$ level, leaving open the possibility that there is additional physics in the Higgs sector. One attractive alternative to the SM is the two Higgs doublet model (2HDM), which has 5 Higgs bosons, allowing for new phenomena in the Higgs sector [3]. The couplings of the Higgs bosons to fermions and gauge bosons in the CP conserving $2 \mathrm{HDM}$ depend on 2 parameters: $\alpha$, which describes the mixing in the neutral Higgs boson sector, and $\tan \beta$, 
the ratio of Higgs vacuum expectation values. Measurements of Higgs coupling properties in the $\mathrm{CP}$ conserving limit require that the $2 \mathrm{HDM}$ be close to the alignment limit, $\beta-\alpha \sim \frac{\pi}{2}[4-7]$.

The SM explains CP violation through the CKM mixing matrix, which is sufficient to account for observed $\mathrm{CP}$ violation in the $B$ and $K$ systems. However, it is insufficient to explain the excess of matter over anti-matter in the universe, suggesting that there may be further sources of $\mathrm{CP}$ violation $[8,9]$. The $2 \mathrm{HDM}$ offers the possibility for a new source of $\mathrm{CP}$ violation beyond the CKM matrix and QCD $\theta$ term. In such a scenario, the $126 \mathrm{GeV}$ Higgs boson can be a mixture of CP even and CP odd states [10-14]. The LHC data has already excluded the case that the 126 Higgs is a pure CP odd scalar $[15,16]$, but the constraints on its CP odd mixture are still rather weak. There have been proposals of new techniques to directly measure the Higgs CP mixture in future colliders [17-24]. The parameters of the $\mathrm{CP}$ violating version of the $2 \mathrm{HDM}$ receive complementary limits from LHC Higgs coupling measurements and from low energy measurements such as electric dipole moments (EDMs). The measurements of Higgs couplings do not put a strong constraint on the $\mathrm{CP}$ violating phase, especially in the alignment limit [10], and the strongest limits come from EDMs [8, 10, 11, 25-27].

$\mathrm{CP}$ violation in the Higgs sector has been studied extensively in the MSSM limit of the 2HDM $[28,29]$. The MSSM contains many sources of CP violation from the soft SUSY breaking terms in the effective Lagrangian [30]. The primary restriction on this type of $\mathrm{CP}$ violation arises from the requirement that the lightest Higgs boson have a mass near $126 \mathrm{GeV}$ [31]. Analogous limits to those obtained in this work from Higgs couplings, heavy Higgs searches, and EDMs can be found in the MSSM [32, 33].

We consider a $\mathrm{CP}$ violating $2 \mathrm{HDM}$ scenario which has a softly broken $Z_{2}$ symmetry which avoids large flavor changing neutral currents from Higgs exchange, but allows for new $\mathrm{CP}$ violation from the scalar potential. We further allow the Higgs couplings to have small deviations from the alignment limit. In this work, we consider the additional constraints on the parameters of the theory arising from the search for heavy Higgs bosons. In the CP conserving $2 \mathrm{HDM}$, the search for heavy Higgs bosons significantly restricts the allowed parameter space for small $\tan \beta[34,35]$ and this remains true in the $\mathrm{CP}$ violating case. In the context of the $2 \mathrm{HDMs}$, if there is significant $\mathrm{CP}$ violation, the heavy Higgs boson masses cannot be too heavy and in some regions of parameter space the LHC heavy Higgs searches can place the leading constraint on $\mathrm{CP}$ violation.

In section 2, we review the CP violating 2HDM and predictions for Higgs boson production and decay within this class of models. Limits from heavy Higgs searches are discussed in section 3 and compared with low energy limits from the electron EDM. We have also updated the results of refs. [10, 36-38] for the limits on the CP violating parameters from Higgs coupling fits. Finally, section 5 contains a concluding discussion of the complementary limits on $\mathrm{CP}$ violating $2 \mathrm{HDM}$ s from Higgs coupling fits, heavy Higgs searches, EDMs, the oblique parameters, and $g-2$. 


\section{Two Higgs doublet models and CP violation}

In this section we review the $2 \mathrm{HDM}$ considered in this study.

\subsection{Scalar potential with two Higgs doublets}

The most general two Higgs doublet potential which breaks $\mathrm{SU}(2)_{L} \times \mathrm{U}(1)$ to $\mathrm{U}(1)_{\mathrm{EM}}$ is,

$$
\begin{aligned}
V\left(\phi_{1}, \phi_{2}\right)= & -\frac{1}{2}\left[m_{11}^{2}\left(\phi_{1}^{\dagger} \phi_{1}\right)+\left(m_{12}^{2}\left(\phi_{1}^{\dagger} \phi_{2}\right)+\text { h.c. }\right)+m_{22}^{2}\left(\phi_{2}^{\dagger} \phi_{2}\right)\right] \\
& +\frac{\lambda_{1}}{2}\left(\phi_{1}^{\dagger} \phi_{1}\right)^{2}+\frac{\lambda_{2}}{2}\left(\phi_{2}^{\dagger} \phi_{2}\right)^{2}+\lambda_{3}\left(\phi_{1}^{\dagger} \phi_{1}\right)\left(\phi_{2}^{\dagger} \phi_{2}\right)+\lambda_{4}\left(\phi_{1}^{\dagger} \phi_{2}\right)\left(\phi_{2}^{\dagger} \phi_{1}\right) \\
& +\frac{1}{2}\left[\lambda_{5}\left(\phi_{1}^{\dagger} \phi_{2}\right)^{2}+\lambda_{6}\left(\phi_{1}^{\dagger} \phi_{2}\right)\left(\phi_{1}^{\dagger} \phi_{1}\right)+\lambda_{7}\left(\phi_{1}^{\dagger} \phi_{2}\right)\left(\phi_{2}^{\dagger} \phi_{2}\right)+\text { h.c. }\right] .
\end{aligned}
$$

The potential of eq. (2.1) leads to tree level flavor changing neutral currents, which can be avoided by imposing a $Z_{2}$ symmetry under which,

$$
\phi_{1} \rightarrow-\phi_{1} \quad \phi_{2} \rightarrow \phi_{2}
$$

Eq. (2.2) implies $\lambda_{6}=\lambda_{7}=0$, while a non-zero $m_{12}$ softly breaks the $Z_{2}$ symmetry of eq. (2.2).

After electroweak symmetry breaking, the Higgs doublets in unitary gauge can be written as,

$$
\phi_{1}=\left(\begin{array}{c}
-\sin \beta H^{+} \\
\frac{1}{\sqrt{2}}\left(v \cos \beta+H_{1}^{0}-i \sin \beta A^{0}\right)
\end{array}\right), \quad \phi_{2}=e^{i \xi}\left(\begin{array}{c}
\cos \beta H^{+} \\
\frac{1}{\sqrt{2}}\left(v \sin \beta+H_{2}^{0}+i \cos \beta A^{0}\right)
\end{array}\right),
$$

where $\tan \beta=v_{2} / v_{1}, v=\sqrt{\left|v_{1}\right|^{2}+\left|v_{2}\right|^{2}}=246 \mathrm{GeV}$ and $H^{+}$is the physical charged Higgs with mass $m_{H^{+}}$. We are free to redefine fields and go to a basis where $\xi=0$. In general there are 2 independent phases and the imaginary parts of $m_{12}$ and $\lambda_{5}$ lead to mixing in the neutral Higgs sector between $H_{1}^{0}, H_{2}^{0}$ and $A^{0}$, and that is the source of CP violation.

The mixing among the three neutral scalars can be parametrized by an orthogonal matrix $R$,

$$
R=\left(\begin{array}{ccc}
-s_{\alpha} c_{\alpha_{b}} & c_{\alpha} c_{\alpha_{b}} & s_{\alpha_{b}} \\
s_{\alpha} s_{\alpha_{b}} s_{\alpha_{c}}-c_{\alpha} c_{\alpha_{c}} & -s_{\alpha} c_{\alpha_{c}}-c_{\alpha} s_{\alpha_{b}} s_{\alpha_{c}} & c_{\alpha_{b}} s_{\alpha_{c}} \\
s_{\alpha} s_{\alpha_{b}} c_{\alpha_{c}}+c_{\alpha} s_{\alpha_{c}} & s_{\alpha} s_{\alpha_{c}}-c_{\alpha} s_{\alpha_{b}} c_{\alpha_{c}} & c_{\alpha_{b}} c_{\alpha_{c}}
\end{array}\right)
$$

where $s_{\alpha}=\sin \alpha$, etc and

$$
-\frac{\pi}{2}<\alpha_{b} \leq \frac{\pi}{2} \quad-\frac{\pi}{2} \leq \alpha_{c} \leq \frac{\pi}{2}
$$

The physical mass eigenstates are then defined as $\left(h_{1}, h_{2}, h_{3}\right)^{T}=R\left(H_{1}^{0}, H_{2}^{0}, A^{0}\right)^{T}$. In the CP conserving version of the $2 \mathrm{HDM}, \alpha_{b}=\alpha_{c}=0, R$ is block diagonal, and $h_{1}$ and $h_{2}$ have no pseudoscalar component. 


\begin{tabular}{|c|c|c|c|c|c|}
\hline & $c_{t, i}$ & $c_{b, i}=c_{\tau, i}$ & $\tilde{c}_{t, i}$ & $\tilde{c}_{b, i}=\tilde{c}_{\tau, i}$ & $a_{i}$ \\
\hline Type I & $R_{i 2} / \sin \beta$ & $R_{i 2} / \sin \beta$ & $-R_{i 3} \cot \beta$ & $R_{i 3} \cot \beta$ & $R_{i 2} \sin \beta+R_{i 1} \cos \beta$ \\
\hline Type II & $R_{i 2} / \sin \beta$ & $R_{i 1} / \cos \beta$ & $-R_{i 3} \cot \beta$ & $-R_{i 3} \tan \beta$ & $R_{i 2} \sin \beta+R_{i 1} \cos \beta$ \\
\hline
\end{tabular}

Table 1. Fermion and gauge boson couplings to Higgs mass eigenstates.

\subsection{Neutral scalar interactions}

For simplicity, we focus on the $2 \mathrm{HDM}$ shere the Yukawa sector has a $Z_{2}$ symmetry and $\phi_{1}$ and $\phi_{2}$ each only gives mass to up or down type fermions. This is sufficient to suppress tree-level flavor changing processes mediated by the neutral Higgs scalars. For the $3 r d$ generation (and suppressing CKM mixing),

$$
\mathcal{L}= \begin{cases}-\left(\frac{\cos \alpha}{\sin \beta} \frac{m_{t}}{v}\right) \bar{Q}_{L}\left(i \tau_{2}\right) \phi_{2}^{*} t_{R}-\left(\frac{\cos \alpha}{\sin \beta} \frac{m_{b}}{v}\right) \bar{Q}_{L} \phi_{2} b_{R}+\text { h.c. } & \text { Type I } \\ -\left(\frac{\cos \alpha}{\sin \beta} \frac{m_{t}}{v}\right) \bar{Q}_{L}\left(i \tau_{2}\right) \phi_{2}^{*} t_{R}+\left(\frac{\sin \alpha}{\cos \beta} \frac{m_{b}}{v}\right) \bar{Q}_{L} \phi_{1} b_{R}+\text { h.c. } & \text { Type II }\end{cases}
$$

where $Q_{L}^{T}=\left(t_{L}, b_{L}\right)$. In both cases, we assume that the charged lepton Yukawa coupling has the same form as that of the charge $-1 / 3$ quarks. Under the $Z_{2}$ symmetry, $Q_{L}, t_{R}, \phi_{2}$ are always even, $\phi_{1}$ is always odd, and $b_{R}$ is even (odd) in Type I (II) models.

From this we can derive the couplings between neutral Higgs bosons and the fermions and gauge bosons. As a general parametrization we take,

$$
\mathcal{L}=\sum_{i=1}^{3}\left[-m_{f}\left(c_{f, i} \bar{f} f+\tilde{c}_{f, i} \bar{f} i \gamma_{5} f\right) \frac{h_{i}}{v}+\left(2 a_{i} M_{W}^{2} W_{\mu} W^{\mu}+a_{i} M_{Z}^{2} Z_{\mu} Z^{\mu}\right) \frac{h_{i}}{v}\right] .
$$

When $c_{f, i} \tilde{c}_{f, i} \neq 0$ or $a_{i} \tilde{c}_{f, i} \neq 0$, the mass eigenstate $h_{i}$ couples to both $\mathrm{CP}$ even and CP odd operators, so the theory violates CP. The coefficients $c_{f, i}, \tilde{c}_{f, i}$ and $a_{i}$ can be derived from $\tan \beta$ and the elements of the matrix $R$ defined above. An appealing feature is that all couplings in table 1 depend on only four parameters, $\alpha, \alpha_{b}, \alpha_{c}$ and $\tan \beta$. It is worth noting that the couplings of the light Higgs boson $h_{1}$ to the gauge bosons and fermions are independent of $\alpha_{c}$. Fits to the CP conserving 2HDM suggest that the couplings are close to the alignment limit, $\beta-\alpha \sim \frac{\pi}{2}$, implying that $h_{1}$ has couplings very close to the SM predictions. In our numerical studies, we will allow small deviations from the alignment limit.

\subsection{CP violation implies a non-decoupled heavy Higgs sector}

In general, the imposed $Z_{2}$ symmetry in the Yukawa sector is not preserved by renormalization. The hard breaking $\lambda_{6}, \lambda_{7}$ terms from the Higgs potential will induce couplings of $\phi_{1}$, $\phi_{2}$ to both up and down type quarks. This does not reintroduce any tree level flavor changing effects because the induced Yukawa matrices are still aligned with the corresponding fermion mass matrices. Motivated by this, a convenient choice is to forbid the $\lambda_{6}, \lambda_{7}$ terms. In this case, the model has an approximate $Z_{2}$ symmetry, which is only softly broken by the $m_{12}^{2}$ term. 
For the approximate $Z_{2}$ symmetric model, all of the potential parameters can be solved for in terms of the following parameters:

- The scalar masses, $m_{h_{1}}, m_{h_{2}}, m_{h_{3}}$ and $m_{H^{ \pm}}$

- The neutral scalar mixing angles, $\alpha, \alpha_{b}, \alpha_{c}$

- The ratio of vev's, $\tan \beta$

- One potential parameter, $\operatorname{Re}\left(m_{12}^{2}\right)$, or $\nu \equiv \operatorname{Re}\left(m_{12}\right)^{2} /\left(v^{2} \sin 2 \beta\right)$,

giving 9 physical parameters. The $\nu$ parameter controls the decoupling limit, i.e., when $\operatorname{Re}\left(m_{12}\right)^{2}$ approaches infinity, the masses of $h_{2}, h_{3}$ and $H^{ \pm}$also go to infinity.

The explicit solution for the parameters of the scalar potential was found in ref. [10], and is listed below in appendix A. The imaginary part of $\lambda_{5}$, which is a source of CP violation, is given by,

$$
\begin{aligned}
\operatorname{Im} \lambda_{5}=\frac{2 \cos \alpha_{b}}{v^{2} \sin \beta}[ & \left(m_{h_{2}}^{2}-m_{h_{3}}^{2}\right) \cos \alpha \sin \alpha_{c} \cos \alpha_{c} \\
& \left.+\left(m_{h_{1}}^{2}-m_{h_{2}}^{2} \sin ^{2} \alpha_{c}-m_{h_{3}}^{2} \cos ^{2} \alpha_{c}\right) \sin \alpha \sin \alpha_{b}\right] .
\end{aligned}
$$

An important point here is that, in order for the $126 \mathrm{GeV}$ Higgs boson to have $\mathrm{CP}$ violating couplings, the heavy Higgs states must not decouple. Otherwise, the two Higgs doublet model will return to the SM limit. This is actually our main motivation for studying the bounds on the non-decoupled heavy Higgs.

Clearly, when the scalars $h_{2,3}$ are much heavier than the electroweak scale, and $m_{h_{2}} \simeq$ $m_{h_{3}} \equiv m_{H^{+}} \gg m_{h_{1}}$

$$
\left|\sin 2 \alpha_{b}\right| \simeq \frac{\left|\operatorname{Im} \lambda_{5}\right| v^{2}}{m_{H^{+}}^{2}}\left|\frac{\sin \beta}{\sin \alpha}\right| .
$$

The unitarity bound on $\operatorname{Im} \lambda_{5}, \operatorname{Im} \lambda_{5}<4 \pi$, sets the largest allowed CP violating mixing angle $\alpha_{b}$. This implies that for an $\mathcal{O}(1) \sin \alpha_{b}$ to be theoretically accessible, the heavy scalars $h_{2}, h_{3}$ and $H^{ \pm}$must be not far above the electroweak scale. In general, for nonzero $\alpha_{b}$, the masses of the heavy scalars should satisfy

$$
m_{H^{+}} \lesssim 870 \mathrm{GeV} \times \sqrt{\left|\operatorname{Im} \lambda_{5}\right| /(4 \pi)} \sqrt{\left|\sin \beta /\left(\sin \alpha \sin 2 \alpha_{b}\right)\right|} .
$$

A similar conclusion holds when one goes beyond the approximate $Z_{2}$ symmetry by including the $\lambda_{6}, \lambda_{7}$ terms.

\subsection{Beyond approximate $Z_{2}$ symmetry}

For the approximate $Z_{2}$ symmetric model, there is a further theoretical constraint on the physical parameters resulting from the minimization of the potential. This constraint is given in eq. (A.10) and can be transformed into a quadratic equation for $\tan \alpha_{c}$. The condition for $\alpha_{c}$ to have a real solution is

$$
\sin ^{2} \alpha_{b} \leq \frac{\left(m_{h_{3}}^{2}-m_{h_{2}}^{2}\right)^{2} \cot ^{2}(\alpha+\beta)}{4\left(m_{h_{2}}^{2}-m_{h_{1}}^{2}\right)\left(m_{h_{3}}^{2}-m_{h_{1}}^{2}\right)} \equiv \sin ^{2} \alpha_{b}^{\max } .
$$


When eq. (2.11) is satisfied, the solutions for $\alpha_{c}$ are,

$$
\alpha_{c}=\left\{\begin{array}{ll}
\alpha_{c}^{-}, & \alpha+\beta \leq 0 \\
\alpha_{c}^{+}, & \alpha+\beta>0
\end{array}, \quad \tan \alpha_{c}^{ \pm}=\frac{\mp\left|\sin \alpha_{b}^{\max }\right| \pm \sqrt{\sin ^{2} \alpha_{b}^{\max }-\sin ^{2} \alpha_{b}}}{\sin \alpha_{b}} \sqrt{\frac{m_{h_{3}}^{2}-m_{h_{1}}^{2}}{m_{h_{2}}^{2}-m_{h_{1}}^{2}}} .\right.
$$

Eq. (2.11) implies an additional theoretical upper bound on the $\mathrm{CP}$ violating angle $\alpha_{b}$, when the other parameters are fixed. In practice, we sometimes find this bound can be stronger than all the experimental limits. However, this is only a bound because of theoretical prejudice. In fact, it can be removed with a minimal step beyond the approximate $Z_{2}$ symmetric case by introducing a $\lambda_{7}$ term, with $\lambda_{7}$ being purely imaginary. In this case, the bound eq. (2.11) no longer exists, $\alpha_{c}$ becomes a free parameter, and $\operatorname{Im} \lambda_{7}$ can in turn be solved for as,

$$
\begin{aligned}
\operatorname{Im} \lambda_{7}=\frac{2 \cos \alpha_{b}}{v^{2} \tan ^{2} \beta}[ & \left(m_{h_{3}}^{2}-m_{h_{2}}^{2}\right) \sin \alpha_{c} \cos \alpha_{c} \frac{\cos (\alpha+\beta)}{\cos ^{2} \beta} \\
& \left.+\left(m_{h_{2}}^{2} \sin ^{2} \alpha_{c}+m_{h_{3}}^{2} \cos ^{2} \alpha_{c}-m_{h_{1}}^{2}\right) \sin \alpha_{b} \frac{\sin (\alpha+\beta)}{\cos ^{2} \beta}\right] .
\end{aligned}
$$

Although introducing hard $Z_{2}$ breaking $\left(\lambda_{6,7} \neq 0\right)$ makes the Yukawa structure in eq. (2.6) unnatural, one might argue it is accidentally the case at the electroweak scale. ${ }^{1}$ In the phenomenological study in the next section, we will give the results for both the approximate $Z_{2}$ case, and the minimal extension as discussed in this subsection.

\subsection{Production and decay of the heavy Higgs at LHC}

\subsubsection{Production}

The dominant heavy Higgs boson production channels relevant to this study are gluon fusion, $g g \rightarrow h_{2,3}$, vector boson fusion, $q q \rightarrow q q h_{2,3}$, and production in association with bottom quarks, $g g / q \bar{q} \rightarrow h_{2,3} b \bar{b}$. In the $2 \mathrm{HDM}$ we explore, the interactions between the heavy neutral Higgs bosons and the SM fermions and the $W, Z$ gauge bosons are simply rescaled from those of a SM-like Higgs boson, $H_{\mathrm{SM}}$, by a factor given in table 1 . Therefore, it is convenient to take the SM-like Higgs cross sections, and rescale them with these factors and the appropriate form factors. The LHC production cross sections for a heavy SM-like Higgs boson have been calculated by the LHC Higgs Cross section Working Group and given in $[42,43]$.

For the gluon fusion process, we calculate the ratio of the heavy Higgs boson production cross section in a $\mathrm{CP}$ violating $2 \mathrm{HDM}$ to that of a SM-like Higgs with the same mass. At one-loop,

$$
R_{g g}^{i}=\frac{\sigma\left(g g \rightarrow h_{i}\right)}{\sigma\left(g g \rightarrow H_{\mathrm{SM}}\right)}=\frac{\left|c_{t, i} A_{1 / 2}^{H}\left(\tau_{t}^{i}\right)+c_{b, i} A_{1 / 2}^{H}\left(\tau_{b}^{i}\right)\right|^{2}+\left|\tilde{c}_{t, i} A_{1 / 2}^{A}\left(\tau_{t}^{i}\right)+\tilde{c}_{b, i} A_{1 / 2}^{A}\left(\tau_{b}^{i}\right)\right|^{2}}{\left|A_{1 / 2}^{H}\left(\tau_{t}^{i}\right)+A_{1 / 2}^{H}\left(\tau_{b}^{i}\right)\right|^{2}},
$$

\footnotetext{
${ }^{1}$ We are aware that allowing $Z_{2}$ breaking terms in the Yukawa sector can introduce additional sources of CP violation. The price for this is introducing tree level flavor changing effects at the same time, and some flavor alignment mechanism must be resorted to [39-41]. We do not consider such a possibility, but focus on CP violation only from the Higgs sector in this work.
} 
where $\tau_{f}^{i}=m_{h_{i}}^{2} /\left(4 m_{f}^{2}\right)$ and $i=1,2,3, f=t, b$. The form factors $A_{1 / 2}^{H}, A_{1 / 2}^{A}$ are given by

$$
\begin{aligned}
A_{1 / 2}^{H}(\tau) & =2(\tau+(\tau-1) f(\tau)) \tau^{-2}, \\
A_{1 / 2}^{A}(\tau) & =2 f(\tau) \tau^{-1}, \\
f(\tau) & =\left\{\begin{array}{ll}
\arcsin ^{2}(\sqrt{\tau}), & \tau \leq 1 \\
\frac{1}{4}\left[\log \left(\frac{1+\sqrt{1-\tau^{-1}}}{1-\sqrt{1-\tau^{-1}}}\right)-i \pi\right]^{2}, & \tau>1
\end{array} .\right.
\end{aligned}
$$

For vector boson fusion, the ratio of the heavy Higgs production cross section in a $2 \mathrm{HDM}$ to that of a SM-like Higgs with the same mass is simply

$$
R_{\mathrm{VBF}}^{i}=\frac{\sigma\left(q q \rightarrow q q h_{i}\right)}{\sigma\left(q q \rightarrow q q H_{\mathrm{SM}}\right)}=\left(a_{i}\right)^{2}
$$

For $h_{2,3} b \bar{b}$ associated production, we take the NLO cross section for SM-like Higgs boson production in the 4 flavor number scheme from refs. [44, 45]. There the cross section contains two pieces, one is proportional to $y_{b}^{2}$, and the other proportional to $y_{b} y_{t}$ from interference. We rescale these results with the heavy Higgs-fermion couplings in a 2HDM,

$$
\sigma\left(b \bar{b} \rightarrow h_{i}\right)=\left(c_{b, i}\right)^{2} \sigma_{b}^{H}\left(m_{h_{i}}\right)+c_{t, i} c_{b, i} \sigma_{t}^{H}\left(m_{h_{i}}\right)+\left(\tilde{c}_{b, i}\right)^{2} \sigma_{b}^{A}\left(m_{h_{i}}\right)+\tilde{c}_{t, i} \tilde{c}_{b, i} \sigma_{t}^{A}\left(m_{h_{i}}\right),
$$

where $\sigma_{b}^{H}$ is the cross section for $g g \rightarrow b \bar{b} h_{i}$ where the Higgs couples to the $b$ quarks, $\sigma_{t}^{H}$ is the interference between diagrams contributing to $g g \rightarrow b \bar{b} h_{i}$ where the Higgs couples to the $b$ and the $t$ quark. $\sigma_{b}^{A}$ and $\sigma_{t}^{A}$ are the corresponding contributions from the pseudoscalar couplings to the $b$ and $t$ quarks given in eq. (2.7). Results in the 5 flavor number scheme [46] are quite similar and do not affect our conclusions.

\subsubsection{Decays}

The heavy neutral scalar to electroweak gauge boson decay rates are

$$
\Gamma\left(h_{i} \rightarrow V V\right)=\left(a_{i}\right)^{2} \frac{G_{F} m_{h_{i}}^{3}}{16 \sqrt{2} \pi} \delta_{V}\left(1-\frac{4 M_{V}^{2}}{m_{h_{i}}^{2}}\right)^{1 / 2}\left[1-\frac{4 M_{V}^{2}}{m_{h_{i}}^{2}}+\frac{3}{4}\left(\frac{4 M_{V}^{2}}{m_{h_{i}}^{2}}\right)^{2}\right],
$$

where $V=W, Z$ and $\delta_{W}=2, \delta_{Z}=1$, and $i=2,3$. We note that in the alignment limit, $\Gamma\left(h_{2,3} \rightarrow V V\right)=0$ when $\sin \alpha_{b}=\sin \alpha_{c}=0$. These channels open up with non-zero CP violation. The decay rates to SM fermions are

$$
\Gamma\left(h_{i} \rightarrow \bar{f} f\right)=\left[\left(c_{f, i}\right)^{2}+\left(\tilde{c}_{f, i}\right)^{2}\right] \frac{N_{c} G_{F} m_{f}^{2} m_{h_{i}}}{4 \sqrt{2} \pi}\left(1-\frac{4 m_{f}^{2}}{m_{h_{i}}^{2}}\right)^{3 / 2},
$$

where $N_{c}=3$ for quarks and 1 for charged leptons.

The heavy scalars can also decay to a pair of gluons via a loop of top or bottom quarks, and the rates are

$\Gamma\left(h_{i} \rightarrow g g\right)=\frac{\alpha_{s}^{2} G_{F} m_{h_{i}}^{3}}{64 \sqrt{2} \pi^{3}}\left[\left|c_{t, i} A_{1 / 2}^{H}\left(\tau_{t}^{i}\right)+c_{b, i} A_{1 / 2}^{H}\left(\tau_{b}^{i}\right)\right|^{2}+\left|\tilde{c}_{t, i} A_{1 / 2}^{A}\left(\tau_{t}^{i}\right)+\tilde{c}_{b}^{i} A_{1 / 2}^{A}\left(\tau_{b}^{i}\right)\right|^{2}\right]$. 
Clearly a decay rate is a $\mathrm{CP}$ even quantity. Thus, in all the above decay rates, the $\mathrm{CP}$ even coefficient $c_{f}^{i}$ and the CP odd one $\tilde{c}_{f}^{i}$ always contribute incoherently.

In our study, we are also interested in the heavy neutral scalars, $h_{2}, h_{3}$, decaying into the $Z$ boson and the $126 \mathrm{GeV}$ Higgs boson,

$$
\begin{aligned}
\Gamma\left(h_{i} \rightarrow Z h_{1}\right)= & \frac{\left|g_{i z 1}\right|^{2}}{16 \pi m_{h_{i}}^{3}} \sqrt{\left(m_{h_{i}}^{2}-\left(m_{h_{1}}+M_{Z}\right)^{2}\right)\left(m_{h_{i}}^{2}-\left(m_{h_{1}}-M_{Z}\right)^{2}\right)} \\
& \times\left[-\left(2 m_{h_{i}}^{2}+2 m_{h_{1}}^{2}-M_{Z}^{2}\right)+\frac{1}{M_{Z}^{2}}\left(m_{h_{i}}^{2}-m_{h_{1}}^{2}\right)^{2}\right],
\end{aligned}
$$

where $g_{i z 1}=\left(e / \sin 2 \theta_{W}\right)\left[\left(-\sin \beta R_{11}+\cos \beta R_{12}\right) R_{i 3}-\left(-\sin \beta R_{i 1}+\cos \beta R_{i 2}\right) R_{13}\right]$.

We have also calculated the decay rate of $h_{i} \rightarrow 2 h_{1}$ from the Higgs self-interactions. The decay rate is

$$
\Gamma\left(h_{i} \rightarrow h_{1} h_{1}\right)=\frac{g_{i 11}^{2} v^{2}}{2 \pi m_{h_{i}}} \sqrt{1-\frac{4 m_{h_{1}}^{2}}{m_{h_{i}}^{2}}},
$$

where $g_{i 11},(i=2,3)$ are defined in appendix B.

To get the branching ratios, we calculate the total width of the heavy Higgs, ${ }^{2}$

$$
\begin{aligned}
\Gamma_{\text {tot }}\left(h_{i}\right)= & \Gamma\left(h_{i} \rightarrow W^{+} W^{-}\right)+\Gamma\left(h_{i} \rightarrow Z Z\right)+\Gamma\left(h_{i} \rightarrow t \bar{t}\right)+\Gamma\left(h_{i} \rightarrow b \bar{b}\right) \\
& +\Gamma\left(h_{i} \rightarrow \tau^{+} \tau^{-}\right)+\Gamma\left(h_{i} \rightarrow g g\right)+\Gamma\left(h_{i} \rightarrow Z h_{1}\right)+\Gamma\left(h_{i} \rightarrow h_{1} h_{1}\right) .
\end{aligned}
$$

Finally, for each channel, the ratio of signal strengths in the 2HDM to the counterpart in the SM is given by,

$$
\mu_{i}^{X X}=\frac{\left(\sigma_{7}^{i} \mathcal{L}_{7}+\sigma_{8}^{i} \mathcal{L}_{8}\right) \times \operatorname{Br}\left(h_{i} \rightarrow X X\right)}{\left(\sigma_{7}^{\mathrm{SM}} \mathcal{L}_{7}+\sigma_{8}^{\mathrm{SM}} \mathcal{L}_{8}\right) \times \operatorname{Br}^{\mathrm{SM}}\left(h_{i} \rightarrow X X\right)},
$$

where, for example, the production cross sections are given by

$$
\sigma_{7}^{i}=\sigma_{g g, 7} R_{g g}^{i}+\sigma_{\mathrm{VBF}, 7} R_{\mathrm{VBF}}^{i}+\sigma_{\mathrm{VH}, 7} R_{\mathrm{VH}}^{i},
$$

$\sigma_{g g, 7}$ is the gluon fusion cross section from refs. [42, 43] for a SM Higgs boson with a mass of $m_{h_{i}}$, and $\mathcal{L}_{7,8}$ are the luminosities used in the experimental analysis. With this quantity, we are able to reinterpret the constraints on a heavy SM-like Higgs boson for the heavy neutral scalars in the 2HDM.

\subsection{CP violation and heavy Higgs signal rates}

At this point, it is useful to gain some intuition about the impact of $\mathrm{CP}$ violation on the heavy Higgs to gauge boson decay channels, $h_{i} \rightarrow V V$ and $h_{i} \rightarrow Z h_{1}$ with $(i=2,3)$. It is convenient to redefine the Higgs doublets and go to a basis where only one doublet, called $\phi_{1}^{\prime}$, gets the $246 \mathrm{GeV}$ vev, while the other $\phi_{2}^{\prime}$ has no vev [13,67].

We start from a special point in the parameter space where the lightest Higgs, $h_{1}$, has exactly the same couplings does the SM Higgs boson. This corresponds to having the mixing angles in eq. (2.4) satisfy $\alpha_{b}=\alpha_{c}=0$, and $\beta-\alpha=\pi / 2$. The Higgs sector preserves

\footnotetext{
${ }^{2}$ The rate $h_{i} \rightarrow \gamma \gamma$ for $i=2,3$ is always small and can be neglected here.
} 

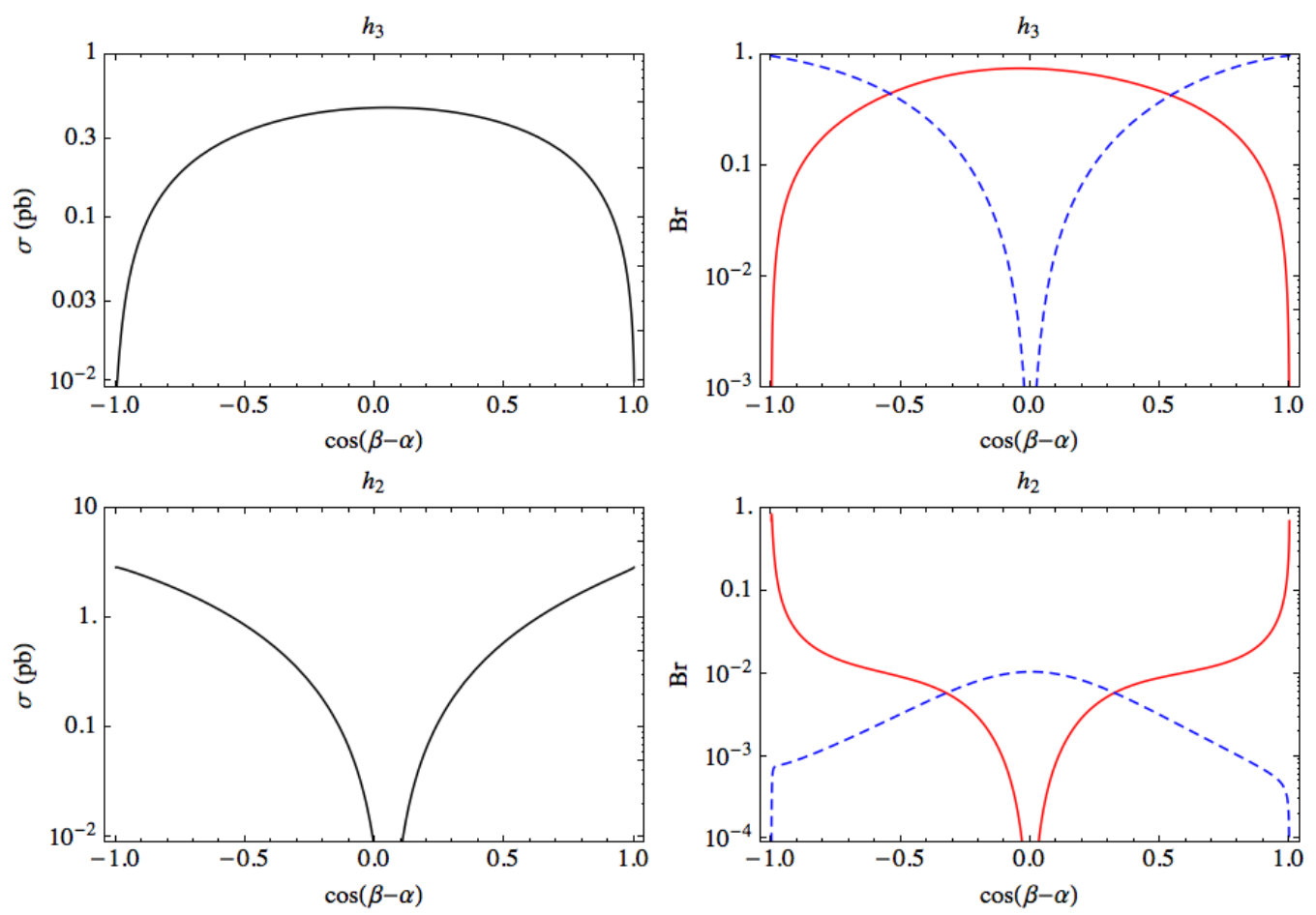

Figure 1. An example showing the impact of a non-zero $\mathrm{CP}$ violating angle, $\alpha_{b}=0.5$, and the deviation from alignment (parameterized by $\cos (\beta-\alpha)$ ) on the heavy Higgs production from gluon fusion at $\sqrt{s}=8 \mathrm{TeV}$ (left panels) and their decays (right panel) in $h_{i} \rightarrow V V$ (red, solid) and $h_{i} \rightarrow Z h_{1}$ (blue, dashed) channels. We have fixed the other parameters to be $\tan \beta=20, \alpha_{c}=0$, $m_{h_{2}}=400 \mathrm{GeV}, m_{h_{3}}=450 \mathrm{GeV}$ and $\nu=1$.

CP invariance at this point. In this case, $h_{1}$ is the excitation arising from $\phi_{1}^{\prime}$ defined above, while $h_{2,3}$ are excitations from $\phi_{2}^{\prime}$. As a result, the decay rates $h_{i} \rightarrow V V$ and $h_{i} \rightarrow Z h_{1}$ both vanish for $i=2,3$. It is worth noticing that this special point can be approached without going to the real decoupling limit by sending the second doublet mass to infinity.

Next, we turn on $\mathrm{CP}$ violation by making $\alpha_{b}=0.5$, but still keep $\alpha_{c}=0$. Here we discuss an example by fixing $\tan \beta=20$ (in the basis of $\left\{\phi_{1}, \phi_{2}\right\}$ given in eq. (2.3)) and vary the angle $\alpha$, or the quantity $\cos (\beta-\alpha)$. We also choose the heavy neutral scalar masses to be $m_{h_{2}}=400 \mathrm{GeV}$ and $m_{h_{3}}=450 \mathrm{GeV}$. In figure 1 , we plot the gluon fusion production cross section and the gauge boson branching ratios of $h_{2,3}$ as a function of $\cos (\beta-\alpha)$. There are several suppressed regions which can be understood from table 1 . In the case $\alpha_{c}=0$, we have in the Type-I model,

$$
\begin{array}{rlrl}
c_{t, 2} & =c_{b, 2}=-\frac{\sin \alpha}{\sin \beta}, & \tilde{c}_{t, 2} & =-\tilde{c}_{b, 2}=0, \\
c_{t, 3} & =c_{b, 3}=-\frac{\cos \alpha}{\sin \beta} \sin \alpha_{b}, & \tilde{c}_{t, 3}=-\tilde{c}_{b, 3}=-\cos \alpha_{b} \cot \beta .
\end{array}
$$

First, the gluon fusion production cross section for $h_{2}$ via a top or bottom loop vanishes at $\alpha=0$ (near $\cos (\beta-\alpha) \simeq 0$ ). In the example we describe here, $\beta=\arctan (20)$ is close to $\pi / 2$, and $c_{t, 2}=c_{b, 2}$ vanishes at $\alpha=0$. Second, at $\alpha= \pm \pi / 2$, (near $\left.\cos (\beta-\alpha) \simeq \pm 1\right)$, the 
couplings $c_{t, 3}$ and $c_{b, 3}$ vanish. As a result, the production cross section for $h_{3}$ is suppressed because $\tilde{c}_{t, 3}$ and $\tilde{c}_{b, 3}$ are both suppressed by $\cot \beta=1 / 20$ in this case. On the other hand, the gauge boson decays of $h_{2,3}$ are directly controlled by $\beta-\alpha$. We list the relevant couplings here, again for $\alpha_{c}=0$,

$$
\begin{array}{ll}
a_{2}=-\cos (\beta-\alpha), & g_{2 z 1}=-\frac{e}{\sin 2 \theta_{W}} \sin (\beta-\alpha) \sin \alpha_{b} \\
a_{3}=-\sin (\beta-\alpha) \sin \alpha_{b}, & g_{3 z 1}=\frac{e}{\sin 2 \theta_{W}} \cos (\beta-\alpha),
\end{array}
$$

where $g_{i z 1}(i=2,3)$ is the coupling between $h_{i}-Z-h_{1}$ defined below eq. (2.23). These make it manifest why the heavy Higgs to gauge boson decay channels are sensitive both to a deviation from the alignment limit and to $\mathrm{CP}$ violation. Clearly, when $\cos (\beta-\alpha)= \pm 1$, the decay rates $h_{3} \rightarrow V V$ and $h_{2} \rightarrow Z h_{1}$ vanish, while when $\cos (\beta-\alpha)=0$, the decay rates $h_{2} \rightarrow V V$ and $h_{3} \rightarrow Z h_{1}$ vanish. For the case of $h_{2}$ decay, the branching ratios are more suppressed because the decay $h_{2} \rightarrow h_{1} h_{1}$ dominates in most of the parameter space. Therefore, the most important constraints come from the $h_{3} \rightarrow V V$ and $h_{3} \rightarrow Z h_{1}$ channels.

Combining eqs. (2.28) and (2.29), we find the $h_{3} \rightarrow V V$ signal rate (production cross section $\times$ decay branching ratio) is peaked at $\cos (\beta-\alpha)=0$, while $h_{3} \rightarrow Z h_{1}$ vanishes at both $\cos (\beta-\alpha)=0, \pm 1$, and is peaked in between. With these facts, one can understand the yellow and orange regions in the upper right panel of figure 11. One can also follow a similar analysis in order to understand the generic features in the other plots.

\section{Results}

In this section, we describe our method to obtain constraints from heavy Higgs searches at the LHC, and show the numerical results in a series of figures.

In the presence of $\mathrm{CP}$ violation, all of the three neutral scalars mix together, and we fix the lightest scalar, $h_{1}$, to be the $126 \mathrm{GeV}$ scalar already discovered at the LHC. As discussed in the previous sections, the heavy Higgs to gauge boson decay channels, including $h_{2,3} \rightarrow W W / Z Z$ and $h_{2,3} \rightarrow Z h_{1} \rightarrow l^{+} l^{-} b \bar{b}$, are not only sensitive to deviations from the alignment limit $(\beta-\alpha=\pi / 2)$, but also to the presence of CP violation $\left(\alpha_{b}, \alpha_{c} \neq 0\right)$. We use the production and decay rates calculated in section 2.5 to obtain the $2 \mathrm{HDM}$ predictions for the heavy Higgs signal strength in these two channels. Then we compare these theory predictions to the results from the 7 and $8 \mathrm{TeV}$ running of the LHC. For the heavy Higgs search data, we use limits for masses up to a TeV from the $h_{2,3} \rightarrow W W / Z Z$ channel $[48,49]$ and from the $h_{2,3} \rightarrow Z h_{1} \rightarrow l^{+} l^{-} b \bar{b}\left(\tau^{+} \tau^{-}\right)$channel [50, 51].

We also take into account the $h_{2,3} \rightarrow \tau^{+} \tau^{-}$channel [52], which gives constraints for heavy Higgs masses up to a $\mathrm{TeV}$ and is relevant in the Type-II model in the large $\tan \beta$ case [53]. The experimental results are given as correlated fits to $\sigma(b \bar{b} \rightarrow h) \operatorname{BR}\left(h \rightarrow \tau^{+} \tau^{-}\right)$ versus $\sigma(g g \rightarrow h) \mathrm{BR}\left(h \rightarrow \tau^{+} \tau^{-}\right)$, which are shown in figure 8 of ref. [52]. We interpret these bounds as bounds on the production of heavy Higgs states in the CP violating 2HDM. For the case $m_{h_{2}}=m_{h_{3}}=300 \mathrm{GeV}$, the limits are shown in figure $2 .{ }^{3}$ In addition to a limit

\footnotetext{
${ }^{3}$ We assume that there is no interference between the $h_{2}$ and $h_{3}$ resonances.
} 


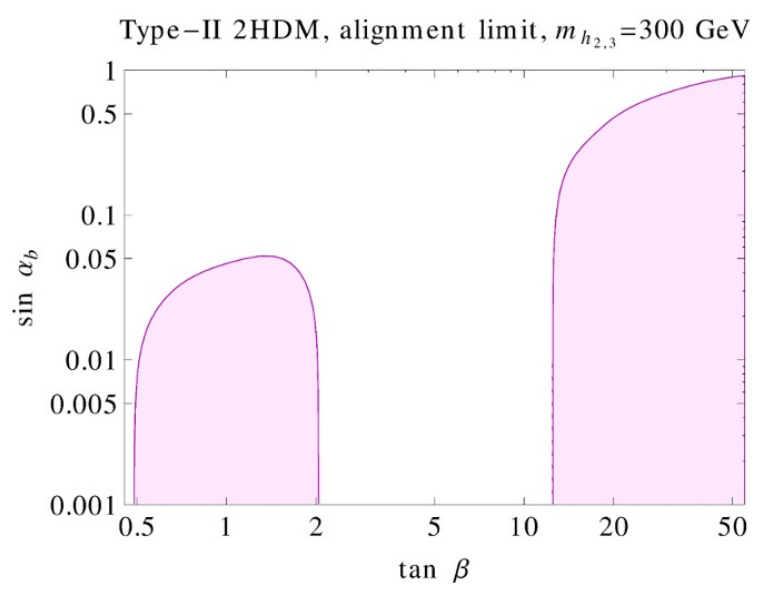

Figure 2. Heavy Higgs search constraints on the Type-II $2 \mathrm{HDM}$ with approximate $Z_{2}$ symmetry and $m_{h_{2}}=m_{h_{3}}=300 \mathrm{GeV}$. The colored regions are excluded by the search for $h_{2,3} \rightarrow \tau^{+} \tau^{-}$.

\begin{tabular}{|l|c|c|c|c|}
\hline Channel & $\mu_{\text {CMS }}$ & Ref. & $\mu_{\text {ATLAS }}$ & Ref. \\
\hline$\mu_{W W}$ & $0.83 \pm 0.21$ & {$[54]$} & $1.09_{-0.21}^{+0.23}$ & {$[55]$} \\
$\mu_{Z Z}$ & $1.0 \pm 0.29$ & {$[54]$} & $1.44_{-0.33}^{+0.40}$ & {$[56]$} \\
$\mu_{\gamma \gamma}$ & $1.13 \pm 0.24$ & {$[54]$} & $1.17 \pm 0.27$ & {$[57]$} \\
$\mu_{b b}$ & $0.93 \pm 0.49$ & {$[54]$} & $0.5 \pm 0.4$ & {$[58]$} \\
$\mu_{\tau \tau}$ & $0.91 \pm 0.27$ & {$[54]$} & $1.4 \pm 0.4$ & {$[59]$} \\
\hline
\end{tabular}

Table 2. ATLAS and CMS Higgs Coupling Measurements.

for $\tan \beta \gtrsim 30$, there is another limit around $\tan \beta \sim 1$. This lower limit arises because the masses of $h_{2,3}$ are below the $t \bar{t}$ threshold and hence the dominant decay channel is through $h_{2,3} \rightarrow b \bar{b}$ and $h_{2,3} \rightarrow \tau^{+} \tau^{-}$. For $m_{h_{2}}=m_{h_{3}}=500 \mathrm{GeV}$ the bound from $h_{2,3} \rightarrow \tau^{+} \tau^{-}$ gives no constraint for $\tan \beta<50$. In general, we find that the bounds from $h_{2,3} \rightarrow \tau^{+} \tau^{-}$ are always weaker than those from the coupling measurement of the light Higgs or EDMs. As a result, we will not include them in the following plots.

The most up-to-date $126 \mathrm{GeV}$ Higgs coupling data are given in table 2, normalized to the appropriate luminosities. They are used to constrain the theoretical predictions for the signal rates of $h_{1}$, from section 2.5. We take the SM cross sections from the LHC Higgs Cross section Working Group [43]. We have performed a $\chi^{2}$ analysis using the results listed in table 2 .

In figures 3 to 12, we show the limits derived from heavy Higgs searches and the light $(126 \mathrm{GeV})$ Higgs data, together with those from the low energy electron and neutron EDMs. For the EDM constraints, we use the results of ref. [10].

In these numerical results, we fix the heavy Higgs masses and the parameter $\nu=1$. The CP violating angle $\alpha_{c}$ is fixed in the approximate $Z_{2}$ symmetric model by eq. (2.12). On the other hand, for the extended model without an approximate $Z_{2}$ symmetry, $\alpha_{c}$ is a free parameter. We also note that varying the parameter $\nu$ between 0 and 1 only leads to slight changes to our results. The constraints are shown in the $\sin \alpha_{b}$ versus $\tan \beta$ plane, while varying $\alpha$ and $\alpha_{c}$. We consider both the alignment limit with $\alpha=\beta-\pi / 2$ and cases 
when there are small deviations from alignment, $\cos (\beta-\alpha)= \pm \Delta$. The $126 \mathrm{GeV}$ Higgs data put upper bounds on $\Delta$ for fixed values of $\tan \beta$. For the Type-I model, we consider $\Delta=0.1$, while for the Type-II model, the light Higgs coupling data constraint is stronger at large $\tan \beta$, so we take $\Delta=0.02$ [4]. ATLAS has also limited the parameters of the $2 \mathrm{HDM}$ by directly searching for the heavier neutral Higgs boson, but these limits are not competitive with the Higgs coupling data for the heavy $h_{2,3}$ masses that we consider [47].

\subsection{Limits from heavy Higgs searches in approximate $Z_{2}$ symmetric models}

We first discuss the models with an approximate $Z_{2}$ symmetry. Figure 3 shows the limits on the CP violating parameter, $\alpha_{b}$, as a function of $\tan \beta$ in the Type-I model. In each panel, the gray area marked "theory inaccessible" has no real solution for $\alpha_{c}$ from eq. (2.12). The left panel assumes the alignment limit, $\beta-\alpha \sim \pi / 2$, while the right panel allows for a small deviation from the alignment limit. ${ }^{4}$ The orange area is excluded by the heavy Higgs search channel $h_{2,3} \rightarrow Z h_{1} \rightarrow l^{+} l^{-} b \bar{b}$, while the yellow area is excluded by the channel $h_{2,3} \rightarrow W W / Z Z$. It is clear that the limits become quite stringent away from the alignment limit. For comparison, we include the results of ref. [10] for the limits from the electron EDM (eEDM, the blue shaded regions are excluded). In all cases, the EDM limit and the heavy Higgs searches exclude complementary regions. The masses of the heavy Higgs are increased to around $600 \mathrm{GeV}$ in figure 4 . In this case, the limits from heavy Higgs searches become much weaker, with the dominant excluded region coming from the eEDM searches. The mass splitting between the heavy masses is restricted by limits on the oblique parameters, which is discussed in section 4.1.

Figure 5 shows the limits on $\alpha_{b}$ versus $\tan \beta$ in the Type-II model. Away from the alignment limit (the right panel), there is a significant exclusion region for $\tan \beta \gtrsim 10$ from the $126 \mathrm{GeV}$ Higgs parameter measurements. Around $\tan \beta \sim 1$, the electron EDM constraint vanishes due to a cancellation among the Barr-Zee diagrams as pointed out in ref. [8]. We find the heavy Higgs searches from the gauge boson decay channels $h_{2,3} \rightarrow$ $Z h_{1}$ and $h_{2,3} \rightarrow W W / Z Z$ are extremely useful and close the window of large values of $\sin \alpha_{b} \sim \mathcal{O}(1)$ in all cases. As the mass of the heavy particles is increased in figure 6 , the region excluded by the heavy Higgs searches shrinks, with again the dominant exclusion coming from the eEDM and neutron EDM (nEDM, the green regions are excluded). It is worth pointing out that the neutron EDM excluded regions are shown using the central values given in [60], which however involves large uncertainties in the evaluation of hadronic matrix elements. In contrast, the heavy Higgs searches provide a robust upper limit on the CP violating angle $\alpha_{b}$.

\subsection{Limits from heavy Higgs searches in the models with no $Z_{2}$ symmetry}

As discussed in section 2.4, if the assumption of an approximate $Z_{2}$ symmetry is relaxed, the theoretical relationship between $\alpha_{b}$ and $\alpha_{c}$ can be removed. In this case $\alpha_{c}$ becomes a free parameter. This helps to remove the theoretically inaccessible region in figures $3-6$, and one can get a complete view of various constraints in the whole parameter space.

\footnotetext{
${ }^{4}$ The results are similar for negative $\Delta$.
} 

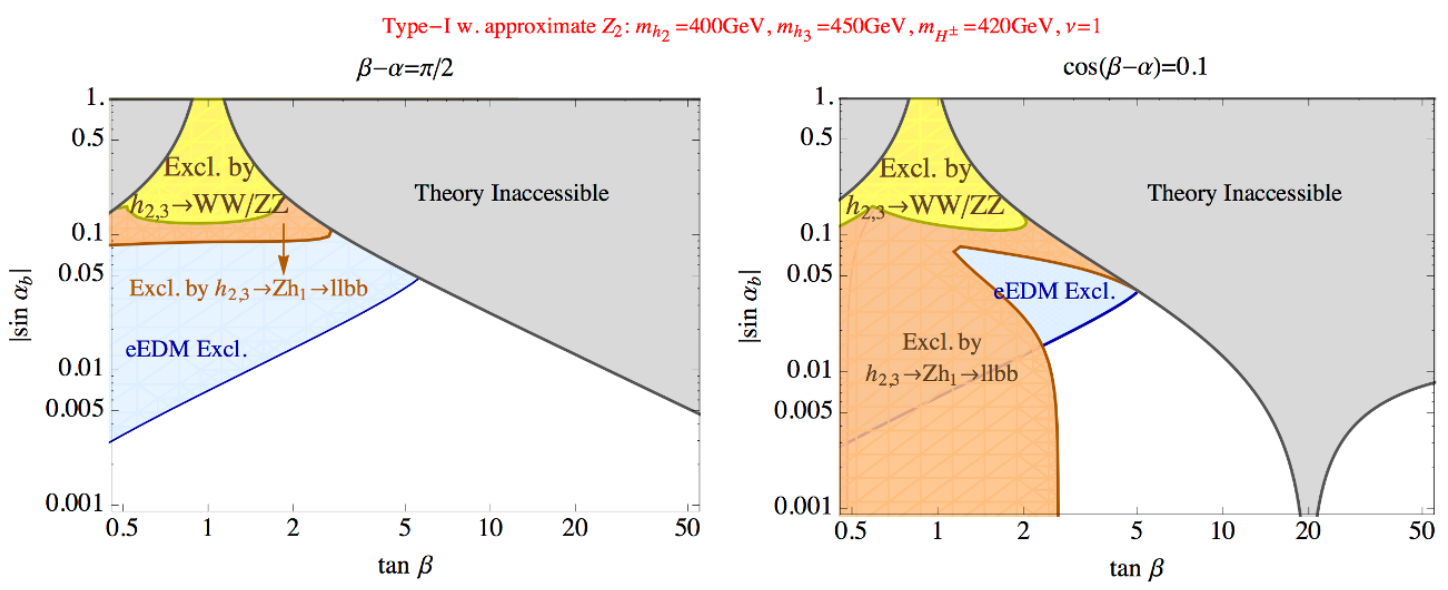

Figure 3. Heavy Higgs search constraints on the Type-I $2 \mathrm{HDM}$ with approximate $Z_{2}$ symmetry, using the $h_{2,3} \rightarrow W W / Z Z$ (yellow) and $h_{2,3} \rightarrow Z h_{1} \rightarrow l^{+} l^{-} b \bar{b}$ (orange) channels. These constraints are presented in the $\sin \alpha_{b}$ versus $\tan \beta$ parameter space and colored regions are excluded. The left panel is for the alignment limit with $\alpha=\beta-\pi / 2$, while the right panel shows the case with a deviation from that limit. Also shown in blue are the electron EDM excluded regions. In these plots, we have chosen the heavy scalar masses to be $m_{h_{2}}=400 \mathrm{GeV}, m_{h_{3}}=450 \mathrm{GeV}, m_{H^{+}}=420 \mathrm{GeV}$, and the model parameter $\nu=1$. The other mixing angle $\alpha_{c}$ is a dependent quantity fixed by eq. (2.12). In the gray region, there is no real solution for $\alpha_{c}$.
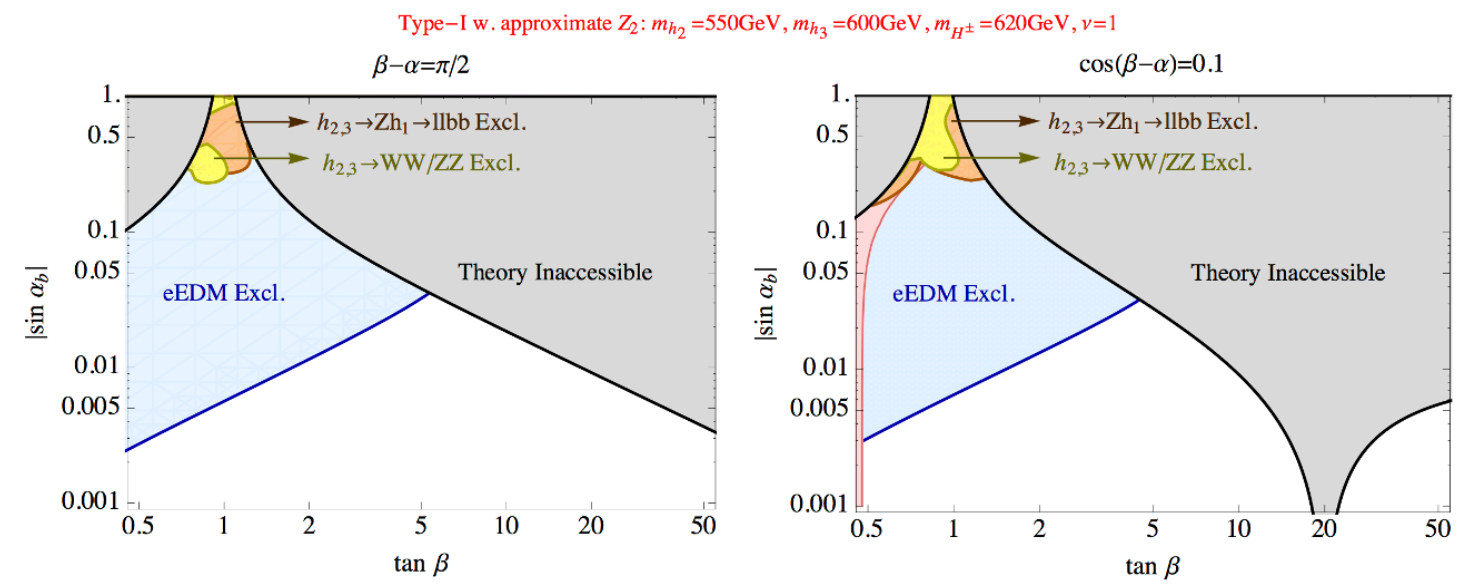

Figure 4. Similar to figure 3 , but with heavy scalar masses $m_{h_{2}}=550 \mathrm{GeV}, m_{h_{3}}=600 \mathrm{GeV}$, $m_{H^{+}}=620 \mathrm{GeV}$. In the right panel, the red region is excluded by the $126 \mathrm{GeV}$ Higgs data applied to $h_{1}$.

Figures 7 and 8 show the constraints in the Type-I model with $\alpha_{c}$ chosen equal to 0 or $\alpha_{b}$, and with two sets of heavy Higgs masses. It is apparent that the dependence on $\alpha_{c}$ is rather weak. The results in the Type-II model are shown in figures 9 and 10. In figures 11 and 12 , the heavy Higgs search constraints are also displayed in the $\alpha_{b}$ and $\cos (\beta-\alpha)$ plane.

It is also worth re-emphasizing that at low $\tan \beta \sim \mathcal{O}(1)$ the $126 \mathrm{GeV}$ Higgs data puts a very weak constraint on the CP violating angle $\alpha_{b}$. This can also be understood 

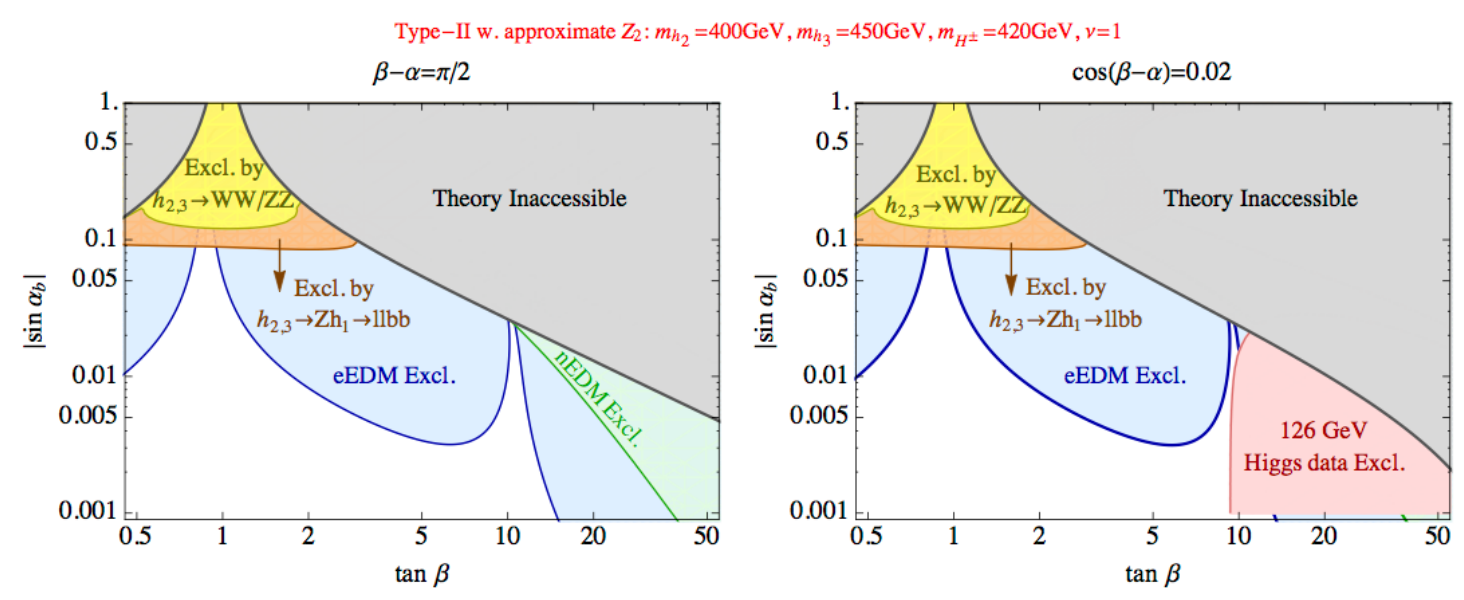

Figure 5. Heavy Higgs search constraints on the Type-II 2HDM with approximate $Z_{2}$ symmetry. The Higgs sector parameters are chosen to be the same as those in figure 3. The colored regions are excluded by searches for $h_{2,3} \rightarrow W W / Z Z$ (yellow), $h_{2,3} \rightarrow Z h_{1} \rightarrow l^{+} l^{-} b \bar{b}$ (orange) channels, $126 \mathrm{GeV}$ Higgs coupling data (red), electron EDM measurements (blue), and neutron EDM limits (green). The gray region is again theoretically excluded because it contains no real solution for $\alpha_{c}$.

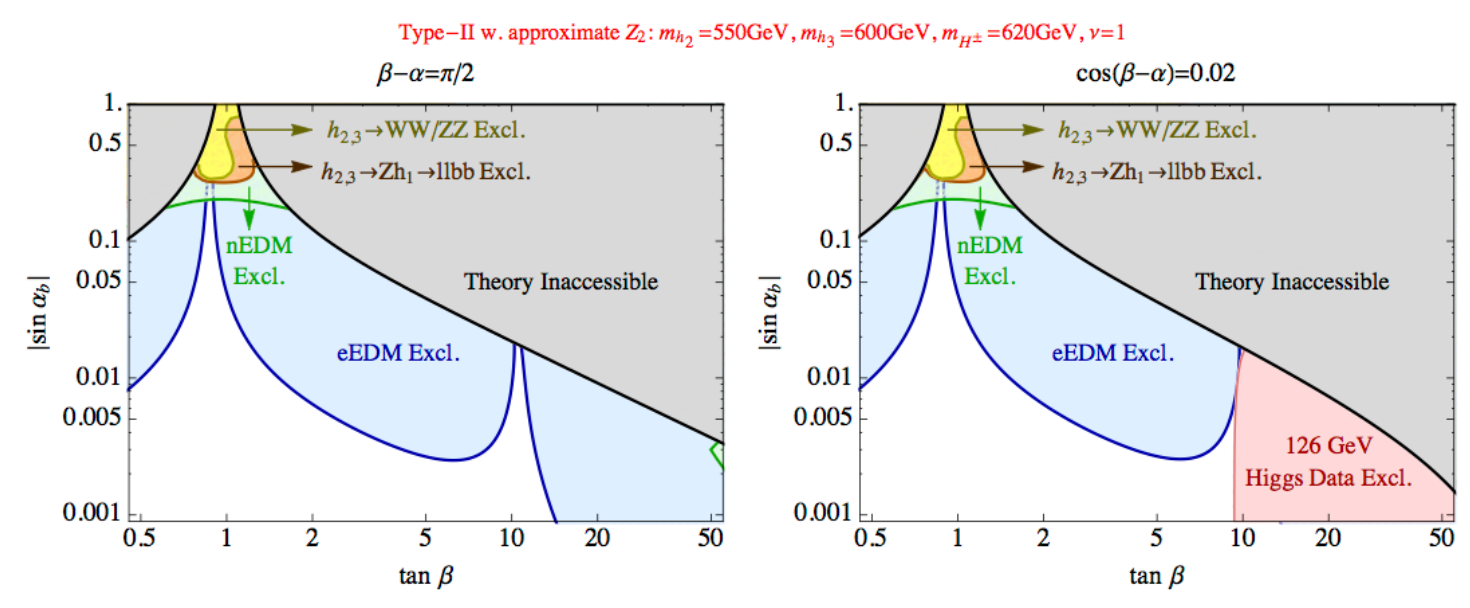

Figure 6. Similar to figure 5 , but with heavy scalar masses $m_{h_{2}}=550 \mathrm{GeV}, m_{h_{3}}=600 \mathrm{GeV}$, $m_{H^{ \pm}}=620 \mathrm{GeV}$.

from table 1, where the lightest $(126 \mathrm{GeV})$ Higgs couplings to other SM particles near the alignment limit are

$$
\begin{aligned}
& a_{1} \simeq \cos \alpha_{b}, \quad c_{t, 1} \simeq(1+\Delta \cot \beta) \cos \alpha_{b}, \quad \tilde{c}_{t, 1} \simeq-\cot \beta \sin \alpha_{b}, \\
& c_{b, 1} \simeq\left\{\begin{array} { l l } 
{ ( 1 + \Delta \operatorname { c o t } \beta ) \operatorname { c o s } \alpha _ { b } , } & { \text { Type-I } } \\
{ ( 1 - \Delta \operatorname { t a n } \beta ) \operatorname { c o s } \alpha _ { b } , } & { \text { Type-II } }
\end{array} \quad \tilde { c } _ { b , 1 } \simeq \left\{\begin{array}{ll}
\cot \beta \sin \alpha_{b}, & \text { Type-I } \\
-\tan \beta \sin \alpha_{b}, & \text { Type-II }
\end{array}\right.\right.
\end{aligned}
$$

where $\Delta=\cos (\beta-\alpha)$ and we have kept terms up to first power in $\Delta$. Clearly for small $\Delta$ and $\tan \beta \approx 1$, all $\mathrm{CP}$ even couplings are approximately $\cos \alpha_{b}$ and all $\mathrm{CP}$ odd couplings $\approx \pm \sin \alpha_{b}$. They approach the values in the SM limit when $\alpha_{b} \rightarrow 0$. In the presence of CP 

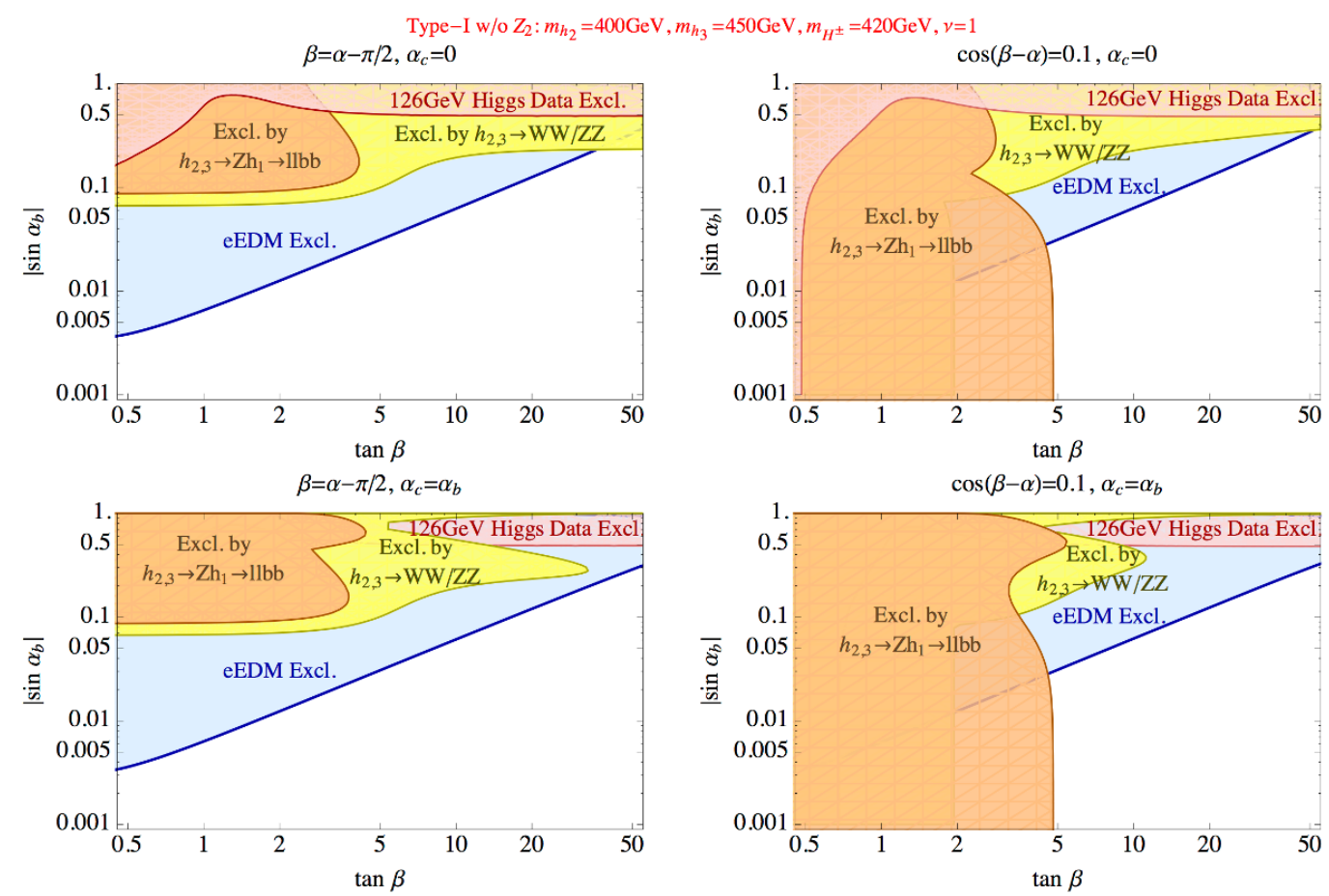

Figure 7. Heavy Higgs search constraints on the Type-I $2 \mathrm{HDM}$ without approximate $Z_{2}$ symmetry, i.e., in this case $\alpha_{c}$ is a free parameter which is allowed to vary. The color scheme for the exclusion regions is the same as in figures $3-6$. The first two rows use the same parameters as figure 3 , and the last two rows use the same as figure 4 .
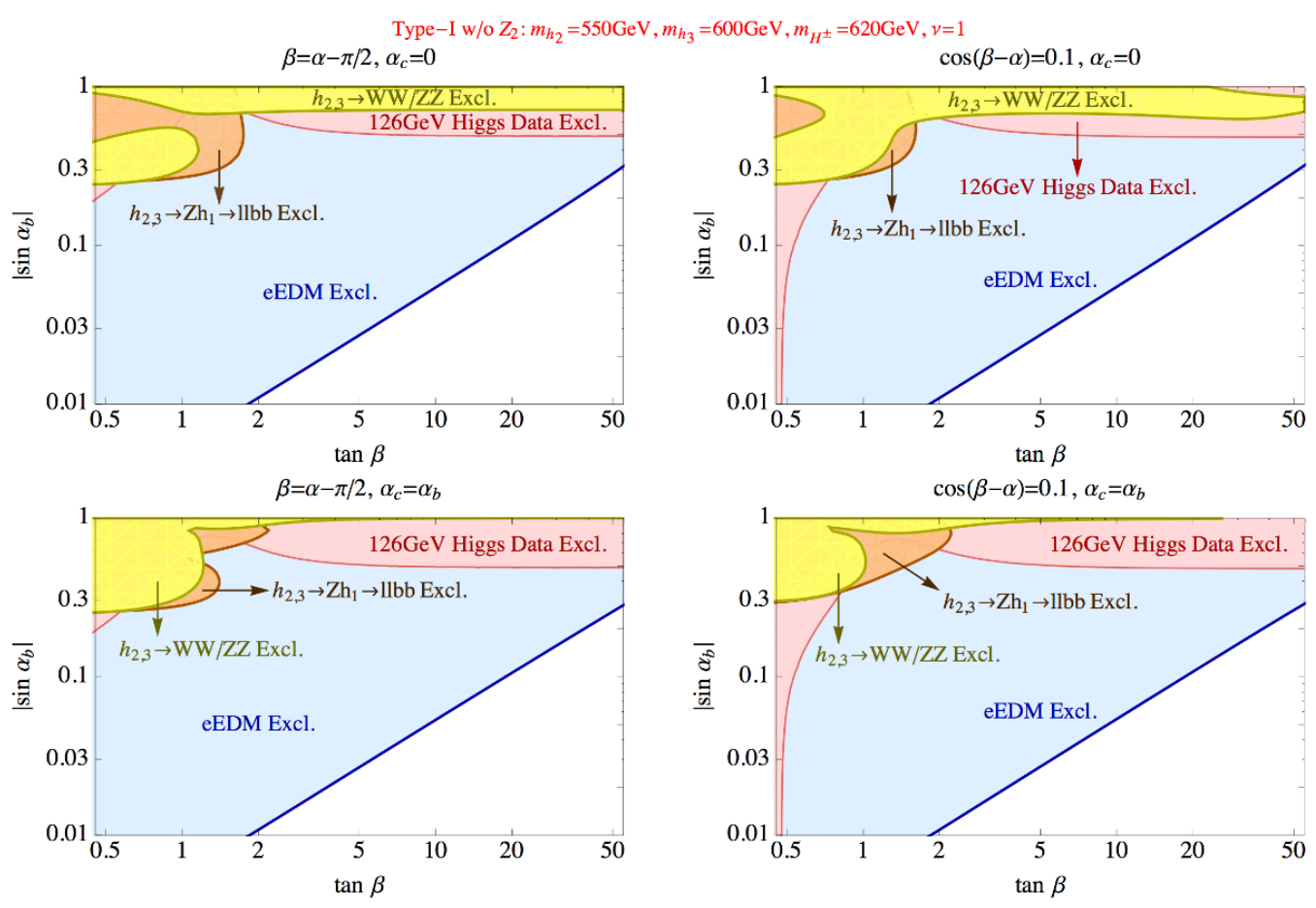

Figure 8. Similar to figure 7, but with heavy Higgs masses $m_{h_{2}}=550 \mathrm{GeV}, m_{h_{3}}=600 \mathrm{GeV}$, $m_{H^{+}}=620 \mathrm{GeV}$. 

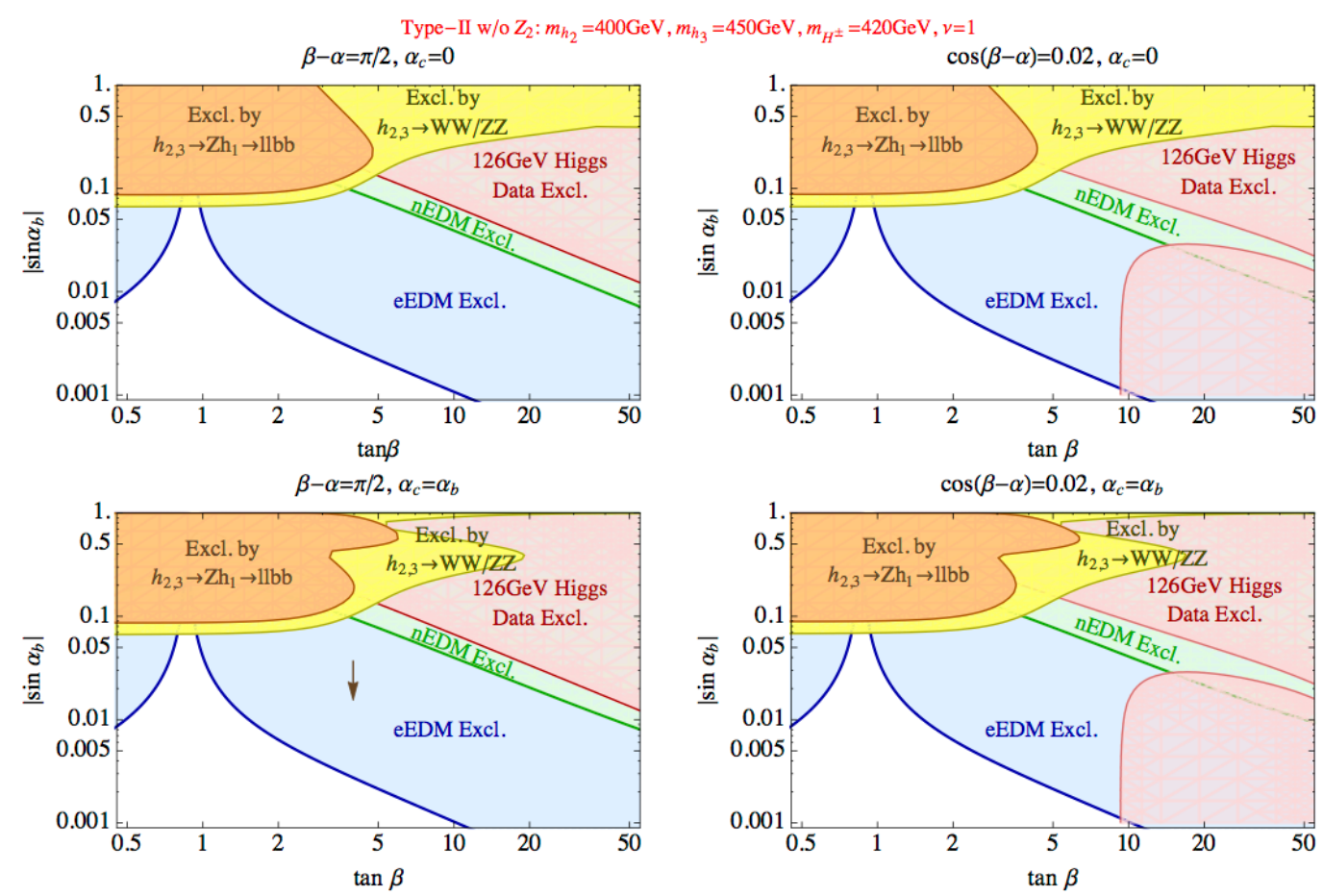

Figure 9. Similar to figure 7, but for the Type-II $2 \mathrm{HDM}$ without approximate $Z_{2}$ symmetry.
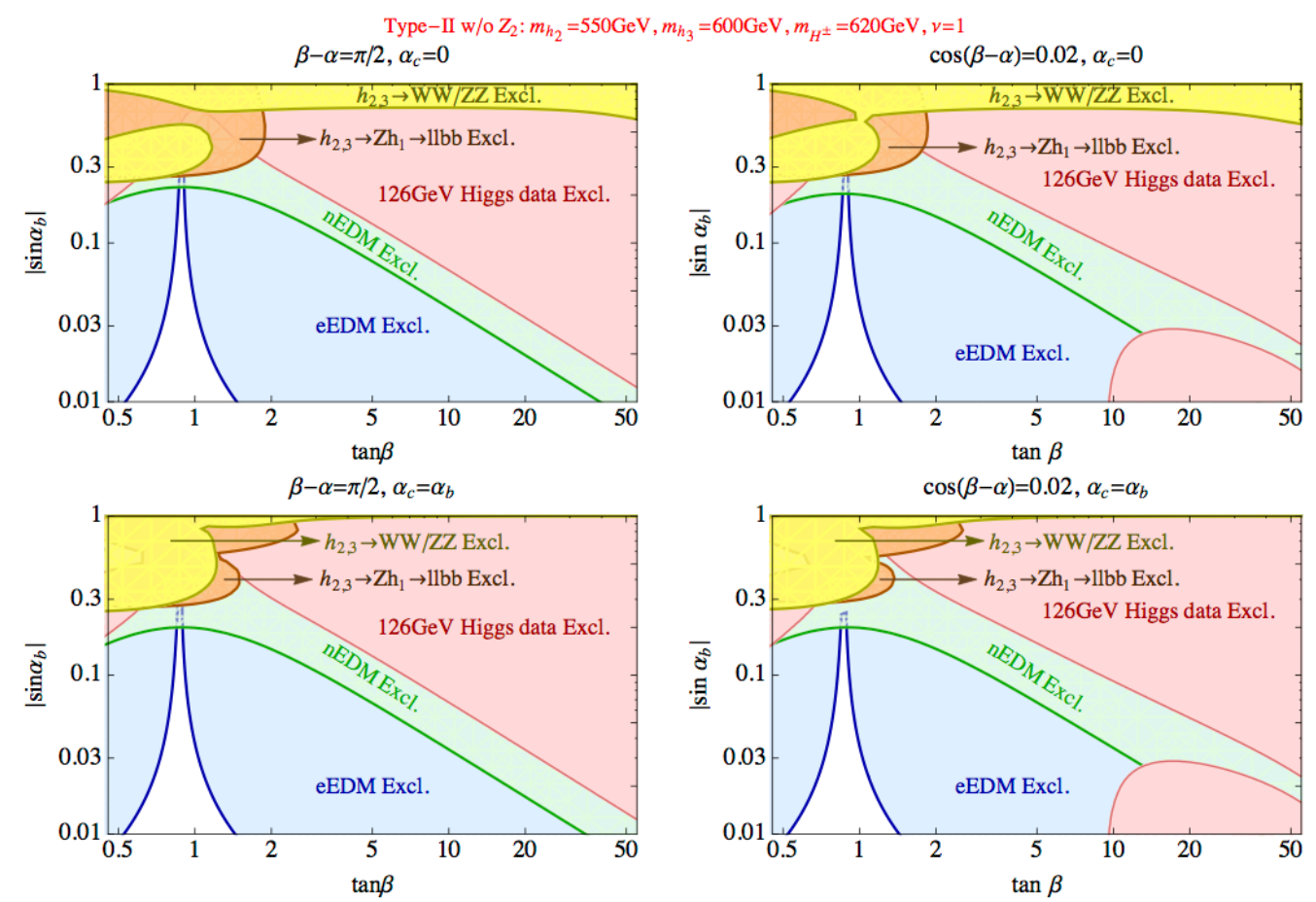

Figure 10. Similar to figure 8 , but for the Type-II $2 \mathrm{HDM}$ without approximate $Z_{2}$ symmetry. 

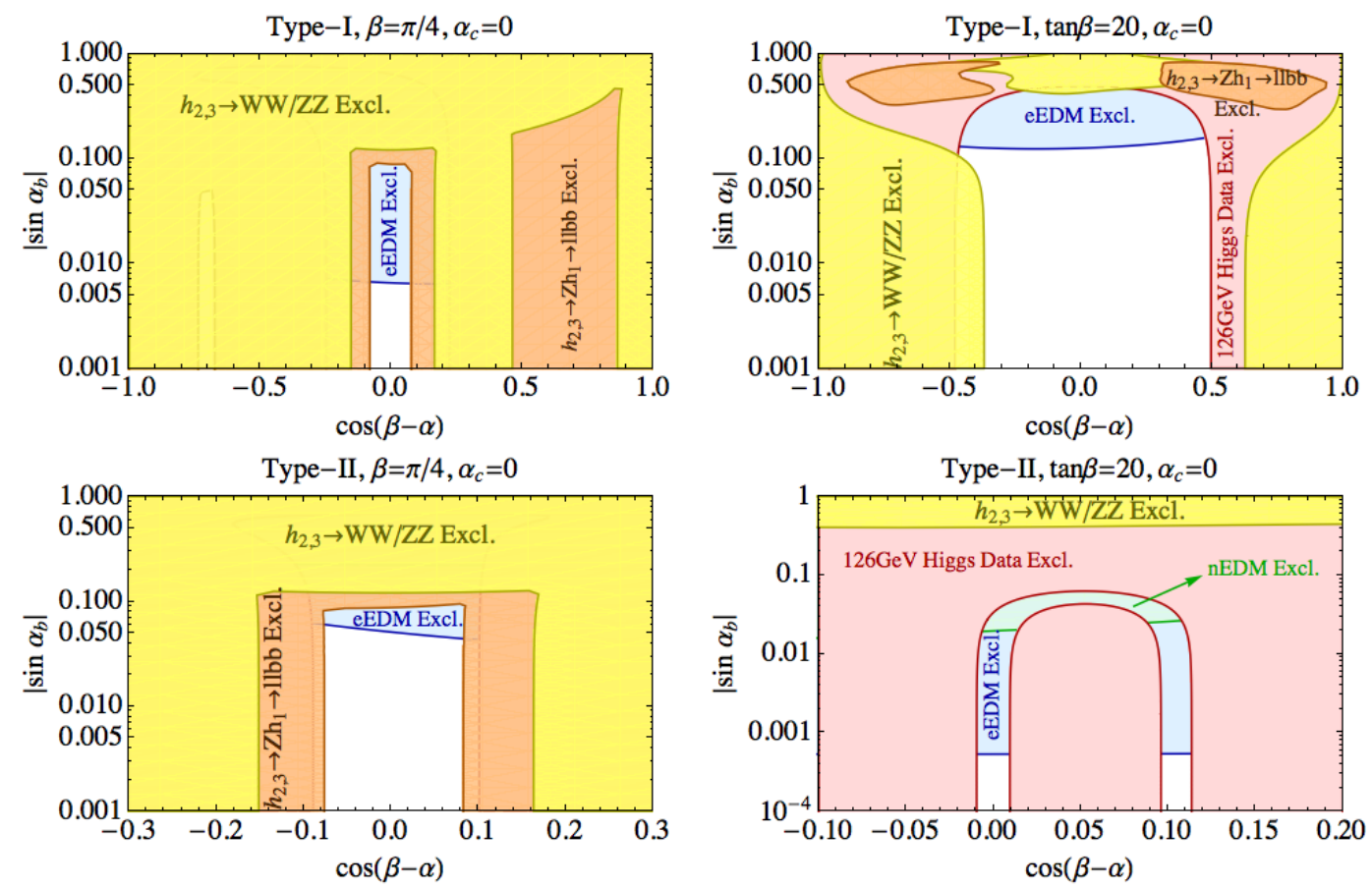

Figure 11. Heavy Higgs search constraints on the Type-I (first row) and Type-II (second row) $2 \mathrm{HDM}$ without approximate $Z_{2}$ symmetry, using the $h_{2,3} \rightarrow W W / Z Z$ (yellow) and $h_{2,3} \rightarrow Z h_{1} \rightarrow$ $l^{+} l^{-} b \bar{b}$ (orange) channels. The heavy scalar masses are fixed to be $m_{h_{2}}=400 \mathrm{GeV}, m_{h_{3}}=450 \mathrm{GeV}$, $m_{H^{+}}=420 \mathrm{GeV}$, and the model parameter $\nu=1$. The other mixing angle $\alpha_{c}=0$.
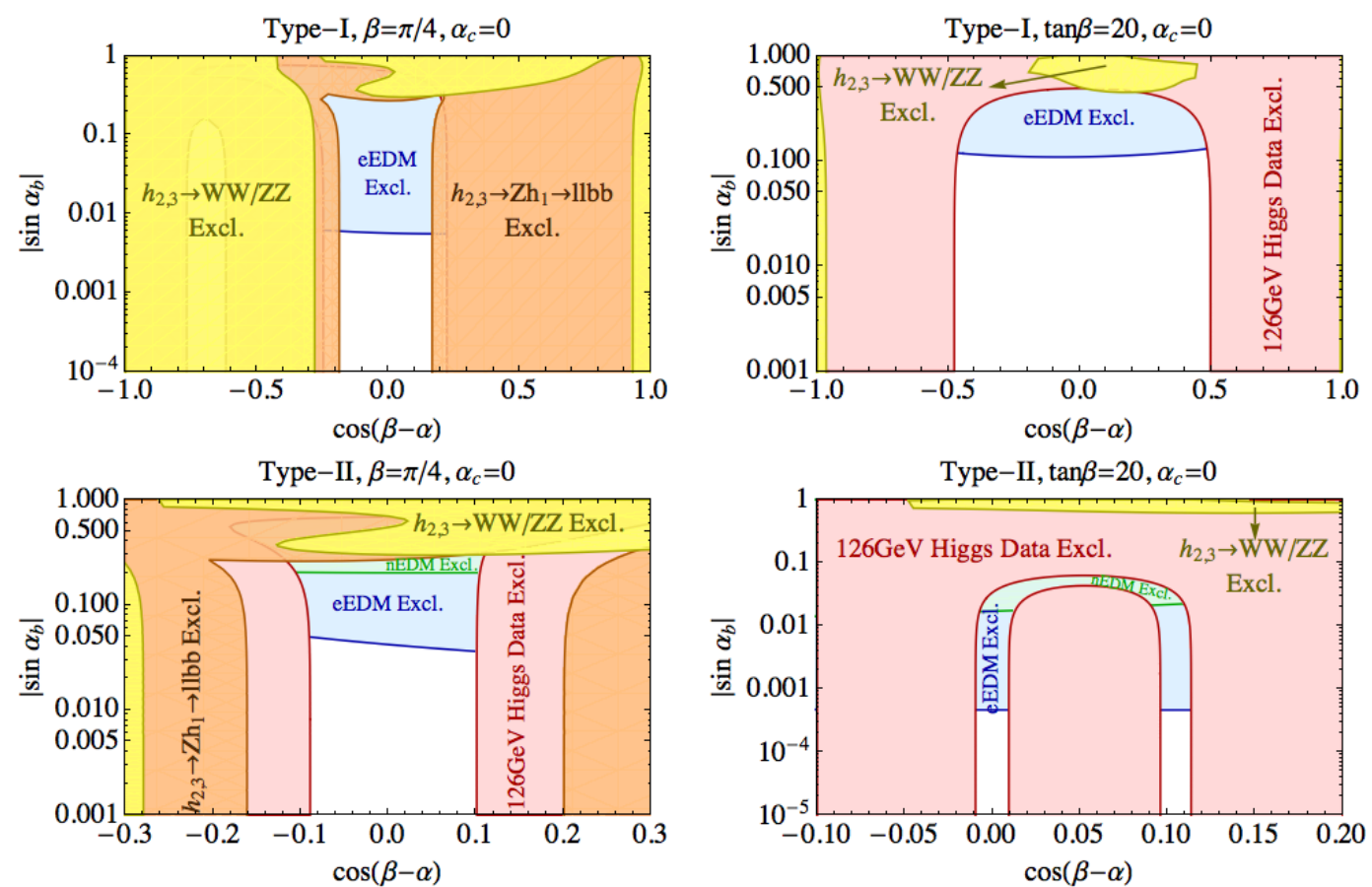

Figure 12. Similar to figure 11, but with heavy scalar masses $m_{h_{2}}=550 \mathrm{GeV}, m_{h_{3}}=600 \mathrm{GeV}$, $m_{H^{+}}=620 \mathrm{GeV}$. 
violation, the gluon fusion production cross section of the light Higgs gets rescaled from the $\mathrm{SM}$ value by a factor $[8,10], \sigma\left(g g \rightarrow h_{1}\right) / \sigma_{\mathrm{SM}}\left(g g \rightarrow H_{\mathrm{SM}}\right) \simeq 1+1.42 \sin ^{2} \alpha_{b}$. The vector boson fusion and associated production rates get suppressed by $\sigma\left(V V \rightarrow h_{1}\right) / \sigma_{\mathrm{SM}}(V V \rightarrow$ $\left.H_{\mathrm{SM}}\right)=\cos ^{2} \alpha_{b}$. The light Higgs to fermion $\left(h_{1} \rightarrow b \bar{b}, \tau^{+} \tau^{-}\right)$decay rates are not affected because the $\mathrm{CP}$ even and $\mathrm{CP}$ odd couplings contribute incoherently, $\Gamma\left(h_{1} \rightarrow f \bar{f}\right) / \Gamma_{\mathrm{SM}}(h \rightarrow$ $f \bar{f})=\cos ^{2} \alpha_{b}+\sin ^{2} \alpha_{b}=1$. The Higgs to gauge boson $\left(h_{1} \rightarrow W W^{*}, Z Z^{*}\right)$ decay rates get suppressed, $\Gamma\left(h_{1} \rightarrow V V^{*}\right) / \Gamma_{\mathrm{SM}}\left(h_{1} \rightarrow V V^{*}\right)=\cos ^{2} \alpha_{b}$. The light Higgs to diphoton decay rate in the presence of $\mathrm{CP}$ violation has been given in refs. $[8,10]$, which in this case can be simplified to $\Gamma\left(h_{1} \rightarrow \gamma \gamma\right) / \Gamma_{\mathrm{SM}}(h \rightarrow \gamma \gamma) \simeq 1-0.81 \sin ^{2} \alpha_{b}$. As a result, the final $\chi^{2}$ of the fit for the $126 \mathrm{GeV}$ Higgs data depends on $\cos ^{2} \alpha_{b}$, and for the SM case $\chi_{\mathrm{SM}}^{2}=\chi^{2}\left(\cos ^{2} \alpha_{b} \rightarrow 1\right)$. Because the $\cos ^{2} \alpha_{b}$ function is very flat near $\alpha_{b}=0$, one can maintain a fit as good as in the SM for sizable $\alpha_{b}$.

In contrast, the heavy Higgs decay to gauge boson channels $\left(h_{2,3} \rightarrow V V\right.$ and $\left.Z h_{1}\right)$ are more sensitive to a non-zero CP violating angle $\alpha_{b}$ and can place a stronger constraint on it. This feature has been discussed in section 2.6. Furthermore, from the figures we notice that at low $\tan \beta$, the heavy Higgs search constraint is stronger than at large $\tan \beta$. This is because $h_{i} \rightarrow t \bar{t},(i=2,3)$ is the dominant decay mode and the branching ratio for the gauge boson decay modes of $h_{i}$ can be written as

$$
\operatorname{Br}_{h_{i} \rightarrow V V \text { or } Z h}(\text { low } \tan \beta) \sim \frac{\Gamma_{h_{i} \rightarrow V V \text { or } Z h}}{\Gamma_{h_{i} \rightarrow t \bar{t}}} .
$$

Eqs. (2.28) and (2.29) tell us that these two rates around the alignment limit are both insensitiveto variations of $\tan \beta$. However, as $\tan \beta$ grows to larger than $\mathcal{O}(2)$, the other decay channels such as $h_{i} \rightarrow h_{1} h_{2}$ and $h_{i} \rightarrow b \bar{b}$ larger than $h_{i} \rightarrow t \bar{t}$, and they are not yet constrained by the LHC data. As a result, the gauge boson decay rates of heavy Higgs bosons are suppressed in this region.

Figure 13 depicts $95 \%$ CL constraints in the $\tan \beta$ versus $\cos (\beta-\alpha)$ plane from heavy Higgs searches (black) and from $126 \mathrm{GeV}$ Higgs data (yellow) on the Type-I (first row) and Type-II (second row) 2HDMs without approximate $Z_{2}$ symmetry. Different curves correspond to $\alpha_{b}=0$ (dotted), 0.1 (solid) and 0.5 (dashed), and the other mixing angle $\alpha_{c}=0$. For the CP conserving case $\left(\alpha_{b}=0\right)$, we found that the bounds are very similar to those studied in refs. [5, 34, 35, 61]. In both Type-I and Type-II models, both heavy and light Higgs searches favor regions around the alignment limit $\cos (\beta-\alpha)=0$. In the Type-II model when CP violation is small (bottom left panel), there is another allowed branch corresponding to $\cos (\beta+\alpha) \sim 0$ [62], but we find the heavy and light Higgs favored regions are inconsistent with each other for very large deviations from the alignment limit. In the Type-I model (first row), the light Higgs bound only depends on $\cos (\beta-\alpha)$, but is independent of $\tan \beta$ in the large $\tan \beta$ limit. The reason is that in this case the $h_{1}$ couplings can be approximated as $c_{t, 1}=c_{b, 1} \rightarrow \sin (\beta-\alpha) \cos \alpha_{b}+\mathcal{O}(1 / \tan \beta), \tilde{c}_{t, 1}=$ $-\tilde{c}_{b, 1}=\mathcal{O}(1 / \tan \beta)$ and $a_{1}=\sin (\beta-\alpha) \cos \alpha_{b}$, so their dependence on $\tan \beta$ is suppressed. On the other hand, for the Type-II model, the couplings $c_{b, 1}, c_{\tau, 1}$ and $\tilde{c}_{b, 1}, \tilde{c}_{\tau, 1}$ are enhanced at large $\tan \beta$. This explains why in the Type-II model (second row), light Higgs data are more restrictive on the parameter space with large $\tan \beta$. As a result, for $\alpha_{b}=0.5$, the light 

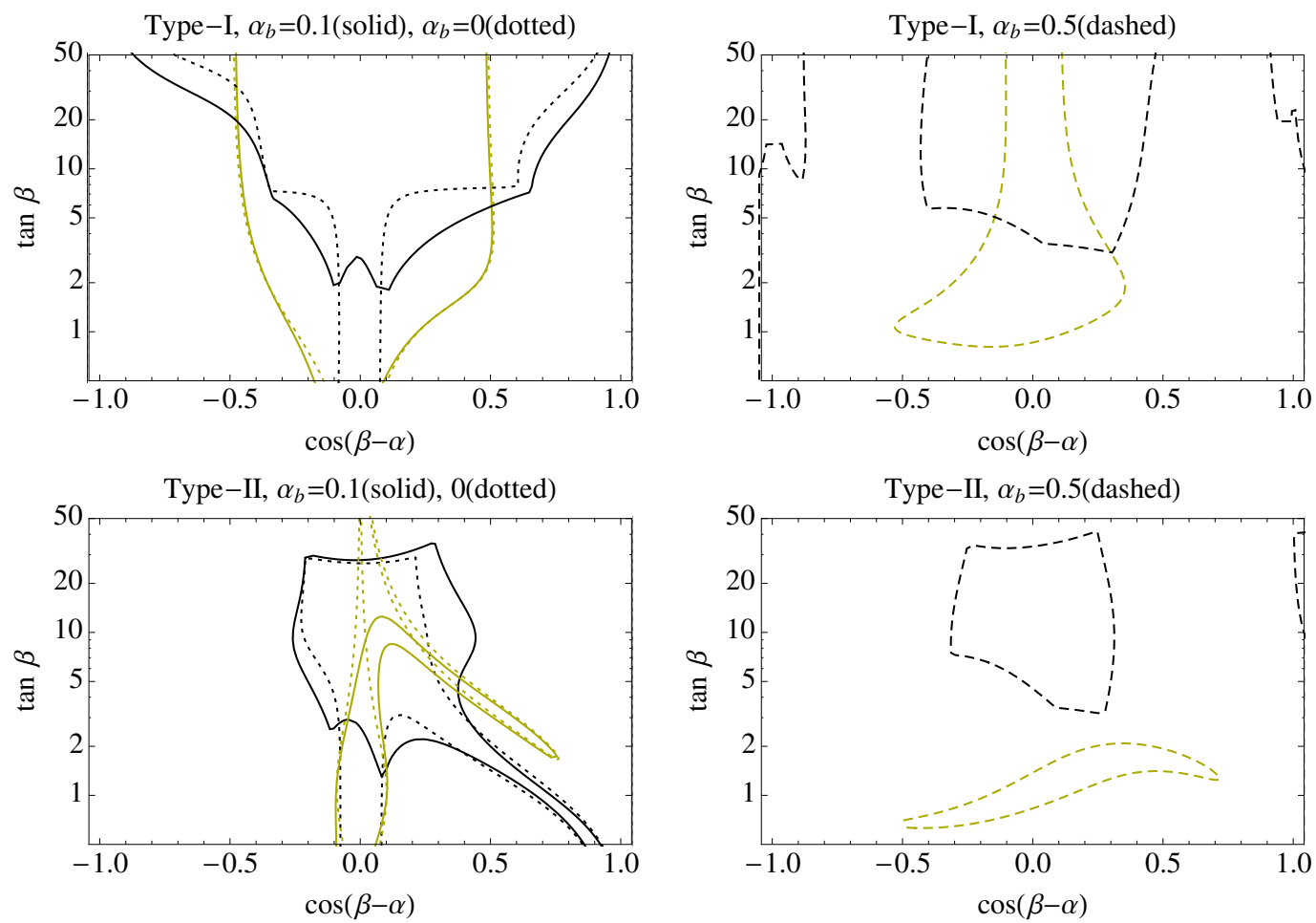

Figure 13. Heavy Higgs search (black) and $126 \mathrm{GeV}$ Higgs data (yellow) constraints at 95\% CL on the Type-I (first row) and Type-II (second row) $2 \mathrm{HDM}$ without approximate $Z_{2}$ symmetry. Different curves correspond to $\alpha_{b}=0$ (dotted), 0.1 (solid) and 0.5 (dashed). The heavy Higgs curves include the combination of constraints from $h_{2,3} \rightarrow W W / Z Z, h_{2,3} \rightarrow Z h_{1} \rightarrow l^{+} l^{-} b \bar{b}$ and $h_{2,3} \rightarrow \tau^{+} \tau^{-}$channels. The heavy scalar masses are fixed to be $m_{h_{2}}=400 \mathrm{GeV}, m_{h_{3}}=450 \mathrm{GeV}$, $m_{H^{ \pm}}=420 \mathrm{GeV}$, and the model parameter $\nu=1$. The other mixing angle $\alpha_{c}=0$.

Higgs data only favors a region with $\tan \beta \lesssim 2$ (see the bottom right panel of figure 13). In contrast, we have learned that the heavy Higgs search data are more sensitive at small $\tan \beta$ and for $\alpha_{b}=0.5$ they only allow the region where $\tan \beta \gtrsim 3$, thus there is no region in the parameter space that can be made consistent with both light and heavy Higgs results from LHC. Figure 14 gives results similar to those in figure 13 but with a different set of mass parameters, $m_{h_{2}}=550 \mathrm{GeV}, m_{h_{3}}=600 \mathrm{GeV}, m_{H^{+}}=620 \mathrm{GeV}$. The parameter space becomes less constrained by the heavy Higgs searches because the production cross sections are smaller compared to those in figure 13.

From the above results, we can conclude that if the heavy Higgs masses lie below around $600 \mathrm{GeV}$, the $\mathrm{CP}$ violating phase $\alpha_{b}$ is constrained to be less than around $30 \%$ throughout the most general parameter space. The regions which allow $\alpha_{b}$ close to this upper bound are $\tan \beta \sim 1$ in the Type-II model, and $\tan \beta \gtrsim 20$ in the Type-I model without an approximate $Z_{2}$ symmetry. We have also estimated the future sensitivity of the heavy Higgs search at the $14 \mathrm{TeV}$ LHC by rescaling the current limits by the square root of expected number of events $(\sigma \times \mathcal{L})$. With $300(3000) \mathrm{fb}^{-1}$ data, if the heavy Higgs 

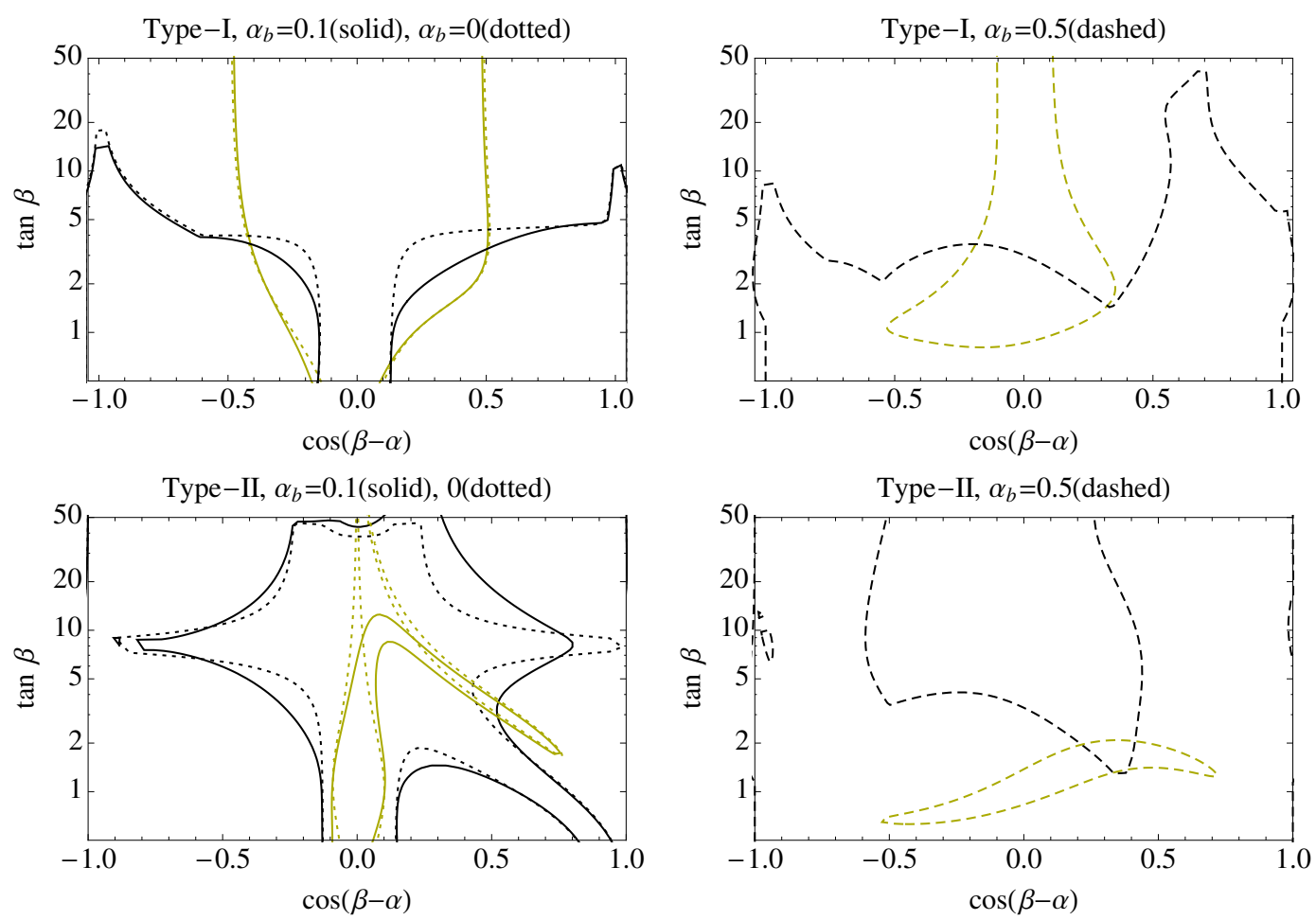

Figure 14. Similar to figure 13, but for heavy Higgs masses $m_{h_{2}}=550 \mathrm{GeV}, m_{h_{3}}=600 \mathrm{GeV}$, $m_{H^{ \pm}}=620 \mathrm{GeV}$.

masses are below $600 \mathrm{GeV}$ and we still do not find them, the $\mathrm{CP}$ violating angle $\alpha_{b}$ will be constrained to be less than around $10 \%$.

Recall that the angle $\alpha_{b}$ parametrizes the size of $\mathrm{CP}$ odd mixture in the $126 \mathrm{GeV}$ Higgs boson. The main point of this work is to show that the heavy Higgs search is relevant and plays a complimentary role to the other indirect searches, and sometimes it stands at the frontier of probing the Higgs boson CP mixture.

\section{Limits from $B$ decays, oblique parameters, and $(g-2)_{\mu}$}

The $\mathrm{CP}$ violating $2 \mathrm{HDM}$ is also limited by measurements in $B$ decays, the oblique parameters, and $(g-2)_{\mu}$. In Type-II models the charged Higgs mass is restricted by $B$ data to be greater than $m_{H^{+}} \sim 340 \mathrm{GeV}$ for all values of $\tan \beta$. In both Type- 1 and Type- 2 models, measurements in the $B$ system prefer $\tan \beta>1[5,63,64]$.

\subsection{Limits from electroweak oblique parameters}

The allowed parameters are restricted by measurements of the oblique parameters. The general results for $S, T$ and $U$ in a $2 \mathrm{HDM}$ are given in refs. [3, 65-67]. In the alignment 
limit, $\cos \alpha=\sin \beta$ and $\sin \alpha=-\cos \beta$, the results simplify considerably,

$$
\begin{aligned}
\alpha \Delta T=\frac{1}{16 \pi^{2} v^{2}}\{ & \sin ^{2} \alpha_{b} F\left(m_{H^{+}}^{2}, m_{h_{1}}^{2}\right)+\left(1-\sin ^{2} \alpha_{b} \sin ^{2} \alpha_{c}\right) F\left(m_{H^{+}}^{2}, m_{h_{2}}^{2}\right) \\
& +\left(1-\sin ^{2} \alpha_{b} \cos ^{2} \alpha_{c}\right) F\left(m_{H^{+}}^{2}, m_{h_{3}}^{2}\right)-\cos ^{2} \alpha_{c} \sin ^{2} \alpha_{b} F\left(m_{h_{1}}^{2}, m_{h_{2}}^{2}\right) \\
& -\sin ^{2} \alpha_{c} \sin ^{2} \alpha_{b} F\left(m_{h_{1}}^{2}, m_{h_{3}}^{2}\right)-\cos ^{2} \alpha_{b} F\left(m_{h_{2}}^{2}, m_{h_{3}}^{2}\right) \\
& +3 \cos ^{2} \alpha_{b}\left[F\left(M_{Z}^{2}, m_{h_{1}}^{2}\right)-F\left(M_{W}^{2}, m_{h_{1}}^{2}\right)\right] \\
& +3 \sin ^{2} \alpha_{c} \sin ^{2} \alpha_{b}\left[F\left(M_{Z}^{2}, m_{h_{2}}^{2}\right)-F\left(M_{W}^{2}, m_{h_{2}}^{2}\right)\right] \\
& +3 \cos ^{2} \alpha_{c} \sin ^{2} \alpha_{b}\left[F\left(M_{Z}^{2}, m_{h_{3}}^{2}\right)-F\left(M_{W}^{2}, m_{h_{3}}^{2}\right)\right] \\
& \left.-3\left[F\left(M_{Z}^{2}, M_{H, \mathrm{ref}}^{2}\right)-F\left(M_{W}^{2}, M_{H, \mathrm{ref}}^{2}\right)\right]\right\}
\end{aligned}
$$

where the last line is the subtraction of the SM Higgs contribution evaluated at the reference scale, $M_{H \text {,ref }}$, at which the fit to the data is performed. The function $F(x, y)$ is,

$$
\begin{aligned}
& F(x, y)=\frac{x+y}{2}-\frac{x y}{(x-y)} \log \left(\frac{x}{y}\right) . \\
& F(x, x)=0, \\
& F(x, y) \stackrel{y \gg x}{\longrightarrow} \frac{y}{2} .
\end{aligned}
$$

With $\alpha_{c}=0$, we obtain the simple form,

$$
\alpha \Delta T=\frac{1}{12 \pi^{2} v^{2}}\left\{\Delta_{2} \Delta_{3} \cos ^{2} \alpha_{b}+\left[\Delta_{1} \Delta_{2}-2\left(\Delta_{3}-\Delta_{1}\right)\left(M_{W}-M_{Z}\right)\right] \sin ^{2} \alpha_{b}\right\}
$$

and $\Delta_{i} \equiv m_{H^{+}}-m_{h_{i}}$. Eq. (4.3) is in agreement with ref. [68] in the limit $\alpha_{b}=0$.

The result for $\Delta S$ also takes a simple form in the alignment limit [65],

$$
\begin{aligned}
\Delta S=\frac{1}{24 \pi}\{ & \cos ^{2} 2 \theta_{W} G\left(m_{H^{+}}^{2}, m_{H^{+}}^{2}, M_{Z}^{2}\right)+\sin ^{2} \alpha_{b}\left[\cos ^{2} \alpha_{c} G\left(m_{h_{1}}^{2}, m_{h_{2}}^{2}, M_{Z}^{2}\right)\right. \\
& \left.+\sin ^{2} \alpha_{c} G\left(m_{h_{1}}^{2}, m_{h_{3}}^{2}, M_{Z}^{2}\right)+\sin ^{2} \alpha_{c} \hat{G}\left(m_{h_{2}}^{2}, M_{Z}^{2}\right)+\cos ^{2} \alpha_{c} \hat{G}\left(m_{h_{3}}^{2}, M_{Z}^{2}\right)\right] \\
& +\cos ^{2} \alpha_{b}\left[\hat{G}\left(m_{h_{1}}^{2}, M_{Z}^{2}\right)+G\left(m_{h_{2}}^{2}, m_{h_{3}}^{2}, M_{Z}^{2}\right)\right]+\ln \left(\frac{m_{h_{1}}^{2} m_{h_{2}}^{2} m_{h_{3}}^{2}}{m_{H^{+}}^{6}}\right) \\
& \left.-\left[\hat{G}\left(M_{H, \text { ref }}^{2}, M_{Z}^{2}\right)+\ln \left(\frac{M_{H, \text { ref }}^{2}}{m_{H^{+}}^{2}}\right)\right]\right\} .
\end{aligned}
$$

Analytic results for $G(x, y, z)$ and $\hat{G}(x, y)$ are given in the appendix of ref. [65].

We use the Gfitter fit to the electroweak data [69],

$$
\begin{aligned}
& S=0.05 \pm 0.11 \\
& T=0.09 \pm 0.13 \\
& U=0.01 \pm 0.11,
\end{aligned}
$$

with a reference value for the SM Higgs mass, $M_{H \text {,ref }}=125 \mathrm{GeV}$. The $S T U$ correlation matrix is,

$$
\rho_{i j}=\left(\begin{array}{ccc}
1 & 0.90 & -0.59 \\
0.09 & 1 & -0.83 \\
-0.59 & -0.83 & 1
\end{array}\right)
$$



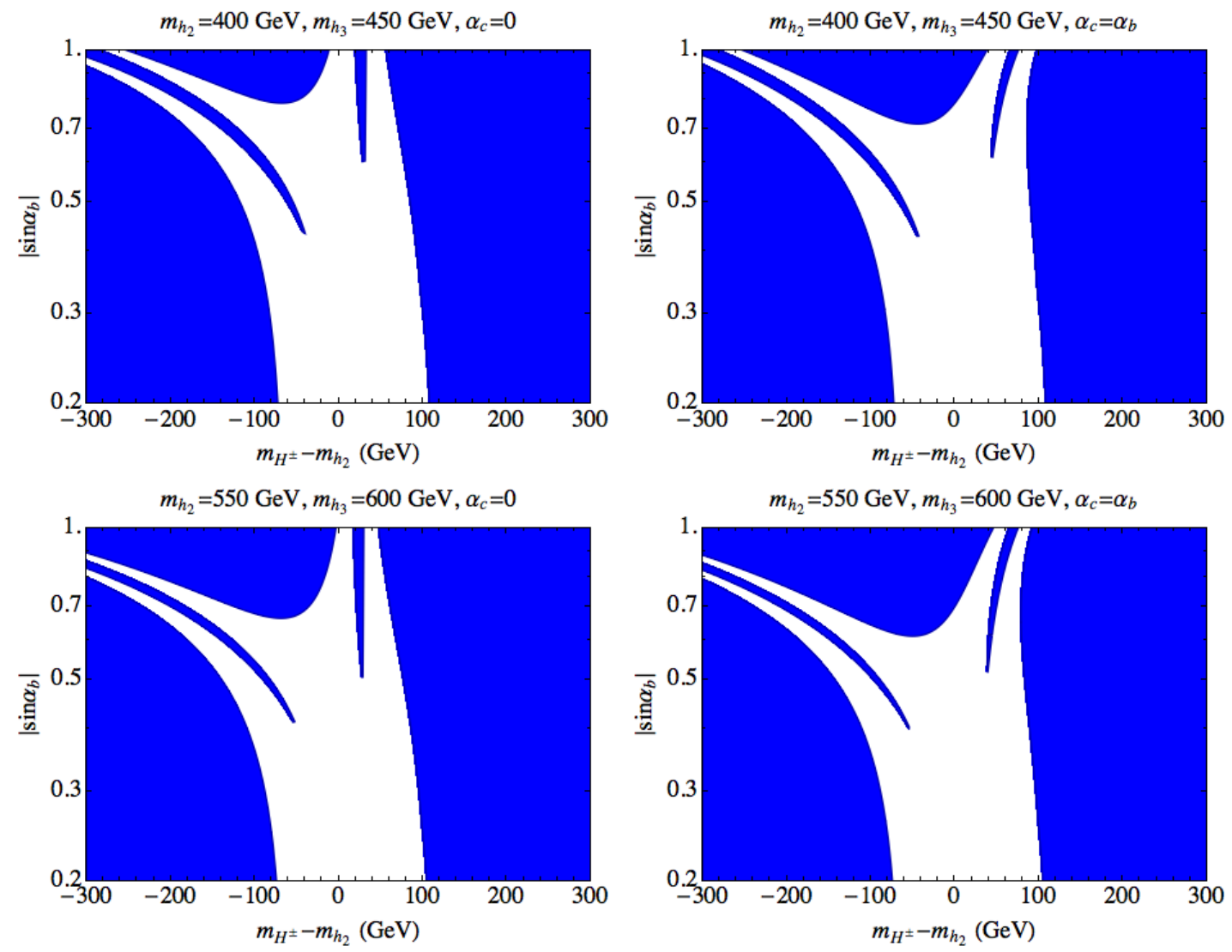

Figure 15. 95\% Confidence Level allowed regions (white) from fits to the oblique parameters in the $\mathrm{CP}$ violating $2 \mathrm{HDM}$.

and the $\chi^{2}$ is defined as

$$
\Delta \chi^{2}=\Sigma_{i j}\left(\Delta X_{i}-\Delta \hat{X}_{i}\right)\left(\sigma^{2}\right)_{i j}^{-1}\left(\Delta X_{j}-\Delta \hat{X}_{j}\right),
$$

where $\hat{X}_{i}=\Delta S, \Delta T$, and $\Delta U$ are the central values of the fit in eq. (4.5), $\hat{X}_{i}=\Delta S, \Delta T$, and $\Delta U$ are the parameters in the $2 \mathrm{HDM}$ (eqs. (4.1) and (4.4)), $\sigma_{i}$ are the errors given in eq. (4.5) and $\sigma_{i j}^{2}=\sigma_{i} \rho_{i j} \sigma_{j}$.

In figure 15 we show the $95 \%$ confidence level allowed regions for $\alpha_{b}=\alpha_{c}$ and $\alpha_{c}=0$. For $\alpha_{b}$ close to 1 , there is some interesting structure due to the interplay of the $\Delta S$ and $\Delta T$ limits. For $\left|\sin \alpha_{b}\right|<0.5$, the results are well approximated by the limit from $\Delta T$ only,

$$
-80 \mathrm{GeV}<\Delta_{2}<120 \mathrm{GeV} .
$$

\subsection{Limits from muon $g-2$}

The experimentally measured value of $\frac{(g-2)_{\mu}}{2}=a_{\mu}$ places a weak constraint on the parameters of the CP violating 2HDM. The deviation between the experimental number and the SM theory prediction is [70],

$$
\Delta a_{\mu}=a_{\mu}^{\exp }-a_{\mu}^{\mathrm{SM}}=265(85) \times 10^{-11} .
$$



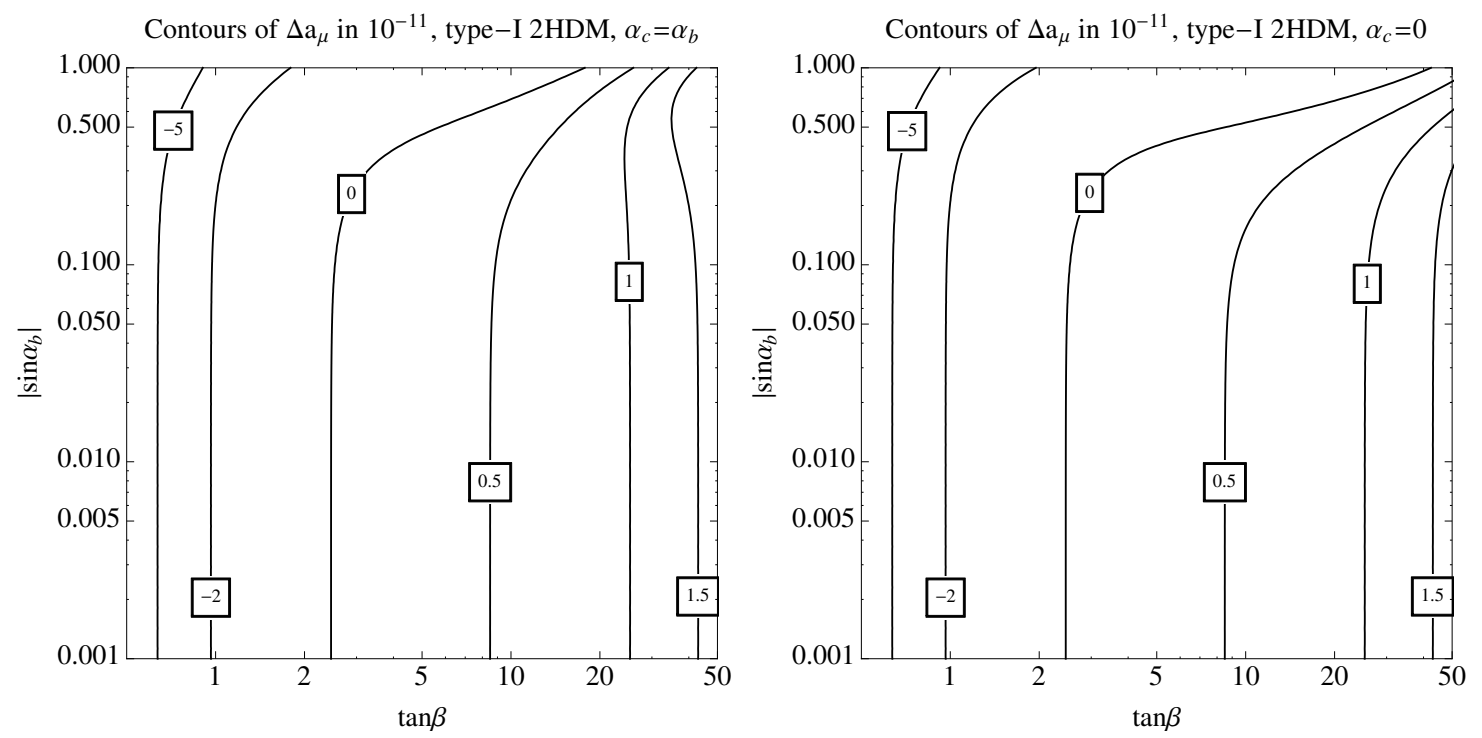

Figure 16. Contributions to $(g-2)_{\mu}$ in the CP violating Type-I $2 \mathrm{HDM}$ from the Barr-Zee diagrams. The heavy scalar masses are fixed to be $m_{h_{2}}=400 \mathrm{GeV}, m_{h_{3}}=450 \mathrm{GeV}, m_{H^{+}}=420 \mathrm{GeV}$, and the model parameter $\nu=1$.
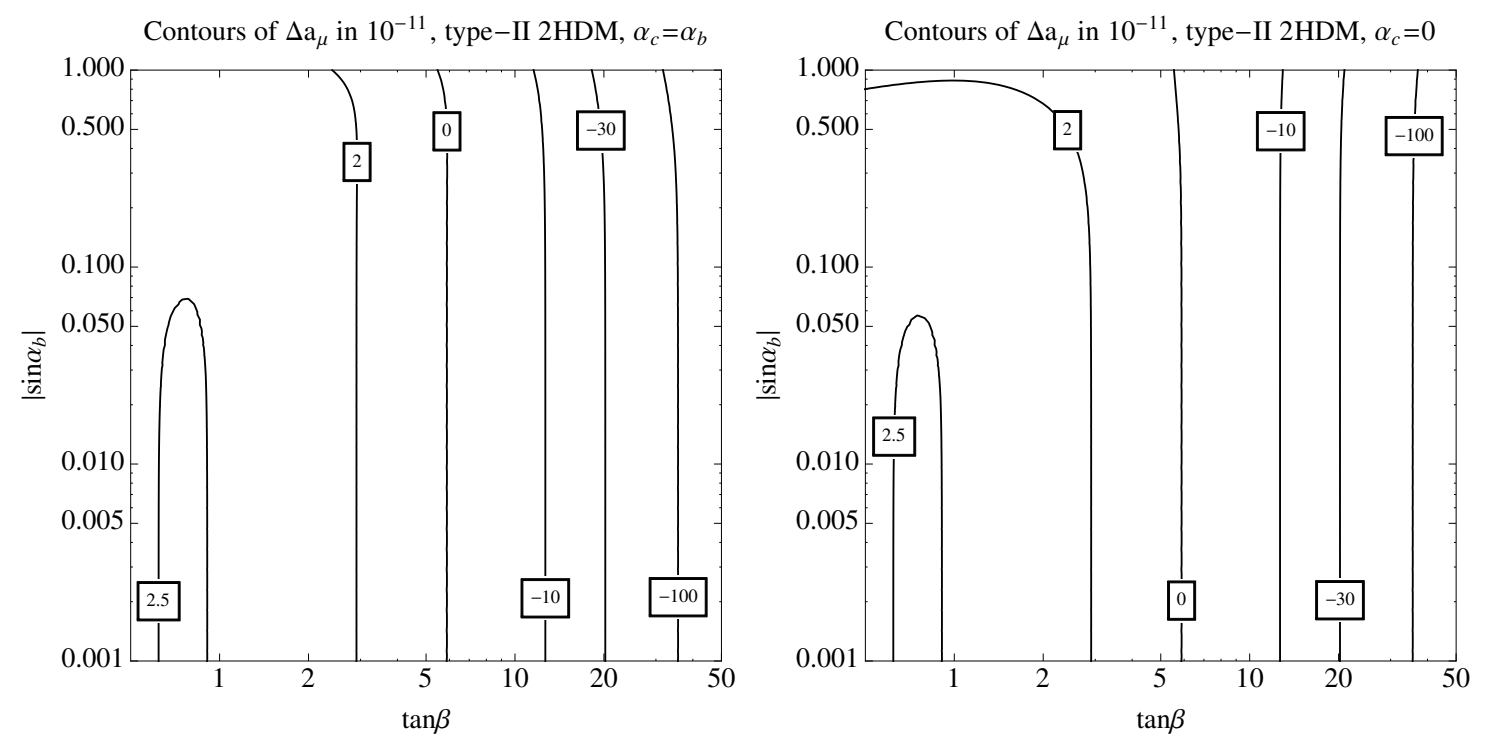

Figure 17. Similar to figure 16 but for Type-II 2HDM.

The one-loop contributions from the Higgs sector in the $2 \mathrm{HDM}$ to $\Delta a_{\mu}$ are numerically small. The larger Higgs sector contributions come from the 2-loop Barr-Zee type diagrams with a closed fermion/gauge-boson/heavy-Higgs loop. This class of diagrams can be enhanced by factors of $M^{2} / m_{\mu}^{2}$ relative to the 1-loop diagrams, where $M$ is a heavy Higgs or heavy fermion mass. For completeness, these results are given in appendix C.

In figures 16 and 17, we show the contributions to $\Delta a_{\mu}$ in the 2HDM for relatively heavy $m_{2,3}$ and $m_{H^{+}}$in units of $10^{-11}$. For $\left|\sin \alpha_{b}\right| \lesssim 0.5$, there is almost no sensitivity to 
the $\mathrm{CP}$ violating phase. The largest contribution is found in the Type-II model for large $\tan \beta$ and is of opposite sign to that needed to explain the discrepancy of eq. (4.9).

\section{Conclusion}

The CP mixture of the $126 \mathrm{GeV}$ Higgs boson is an important property of the Higgs sector that deserves further scrutiny. A non-zero CP component is theoretically well motivated and may be the origin of the cosmic baryon asymmetry. An important consequence of the $126 \mathrm{GeV}$ Higgs boson having a sizable CP odd mixture is that the new physics responsible for this cannot be decoupled and must lie near the electroweak scale.

In the context of $\mathrm{CP}$ violating, flavor conserving two-Higgs-doublet models, we studied the impact of the heavy Higgs searches at the LHC on the CP violating parameters. In this class of models, CP violation appears in the neutral Higgs sector, where there are two more real scalars $\left(h_{2,3}\right)$ in addition to the lightest $126 \mathrm{GeV}$ one. The couplings of the heavy Higgs scalars with electroweak gauge bosons are very sensitive to the $\mathrm{CP}$ violation in the Higgs sector. Turning on a CP odd mixture in the $126 \mathrm{GeV}$ Higgs boson will also turn on the heavy Higgs decay channels into gauge bosons, $h_{2,3} \rightarrow W W / Z Z$ and $Z h_{1}$. There is data from the LHC from the search for a SM like Higgs boson in these decay channels, and the non-discovery of a heavy Higgs can be re-interpreted as constraints on the allowed deviation from the alignment limit in the two-Higgs-doublet models without $\mathrm{CP}$ violation.

In this work, we point out that heavy Higgs searches are also extremely useful for constraining Higgs sector CP violation and in particular the CP mixture of the $126 \mathrm{GeV}$ Higgs boson. We demonstrate that the constraints from heavy Higgs searches are largely complimentary to the low energy EDM constraints. We compare our results with the limits from the global fit to the $126 \mathrm{GeV}$ Higgs data, and find they can place much stronger limits than the light Higgs coupling fit, especially in the interesting regions when there are destructive contributions to the EDM. We find in these regions that the heavy Higgs searches are at the frontier of probing Higgs sector CP violation. The current limit on the $\mathrm{CP}$ violating mixing angle, parametrized by $\alpha_{b}$, is constrained to be less than $30 \%$, and the LHC heavy Higgs search can further narrow down the angle to less than a $10 \%$ level with the high luminosity runs. We also expect our work to be a roadmap for the future searches for Higgs sector CP violation and the exciting interplay across various experimental frontiers.

For completeness, we have also explored other relevant constraints from electroweak oblique parameters, the muon $g-2$ and from B physics, and discussed their implications on the heavy Higgs parameter limits in CP violating 2HDMs. 


\section{A Solving the potential parameters in the approximate $Z_{2}$ case}

In this section, we list the relations between the potential parameters and the phenomenological parameters listed in eq. (2.3) in the approximate $Z_{2}$ symmetric $2 \mathrm{HDMs}$.

$$
\begin{aligned}
& m_{11}^{2}=\lambda_{1} v^{2} \cos ^{2} \beta+\left(\lambda_{3}+\lambda_{4}\right) v^{2} \sin ^{2} \beta-\operatorname{Re}\left(m_{12}^{2} e^{i \xi}\right) \tan \beta+\operatorname{Re}\left(\lambda_{5} e^{2 i \xi}\right) v^{2} \sin ^{2} \beta \\
& m_{22}^{2}=\lambda_{2} v^{2} \sin ^{2} \beta+\left(\lambda_{3}+\lambda_{4}\right) v^{2} \cos ^{2} \beta-\operatorname{Re}\left(m_{12}^{2} e^{i \xi}\right) \cot \beta+\operatorname{Re}\left(\lambda_{5} e^{2 i \xi}\right) v^{2} \cos ^{2} \beta \\
& \operatorname{Im}\left(m_{12}^{2}\right)=v^{2} \sin \beta \cos \beta \operatorname{Im}\left(\lambda_{5}\right) \\
& \lambda_{1}=\frac{m_{h_{1}}^{2} \sin ^{2} \alpha \cos ^{2} \alpha_{b}+m_{h_{2}}^{2} R_{21}^{2}+m_{h_{3}}^{2} R_{31}^{2}}{v^{2} \cos \beta^{2}}-\nu \tan ^{2} \beta \\
& \lambda_{2}=\frac{m_{h_{1}}^{2} \cos ^{2} \alpha \cos ^{2} \alpha_{b}+m_{h_{2}}^{2} R_{22}^{2}+m_{h_{3}}^{2} R_{32}^{2}}{v^{2} \sin \beta^{2}}-\nu \cot ^{2} \beta \\
& \lambda_{4}=2 \nu-\operatorname{Re}_{5}-\frac{2 m_{H^{+}}^{2}}{v^{2}}, \\
& \lambda_{3}=\nu-\frac{m_{h_{1}}^{2} \sin ^{2} \cos _{\alpha} \cos ^{2} \alpha_{b}-m_{h_{2}}^{2} R_{21} R_{22}-m_{h_{3}}^{2} R_{31} R_{32}}{v^{2} \sin _{\beta} \cos \beta}-\lambda_{4}-\operatorname{Re} \lambda_{5} \\
& \operatorname{Re} \lambda_{5}=\nu-\frac{m_{h_{1}}^{2} \sin ^{2} \alpha_{b}+\cos ^{2} \alpha_{b}\left(m_{h_{2}}^{2} \sin ^{2} \alpha_{c}+m_{h_{3}}^{2} \cos ^{2} \alpha_{c}\right)}{v^{2}} \\
& \operatorname{Im} \lambda_{5}=\frac{2 \cos \alpha_{b}}{v^{2} \sin \beta}\left[\left(m_{h_{2}}^{2}-m_{h_{3}}^{2}\right) \cos ^{2} \sin _{c} \cos \alpha_{c}\right. \\
&\left.\quad+\left(m_{h_{1}}^{2}-m_{h_{2}}^{2} \sin ^{2} \alpha_{c}-m_{h_{3}}^{2} \cos ^{2} \alpha_{c}\right) \sin \alpha \sin \alpha_{b}\right]
\end{aligned}
$$

There is an additional constraint,

$$
\tan \beta=\frac{\left(m_{h_{2}}^{2}-m_{h_{3}}^{2}\right) \cos \alpha_{c} \sin \alpha_{c}+\left(m_{h_{1}}^{2}-m_{h_{2}}^{2} \sin ^{2} \alpha_{c}-m_{h_{3}}^{2} \cos ^{2} \alpha_{c}\right) \tan \alpha \sin \alpha_{b}}{\left(m_{h_{2}}^{2}-m_{h_{3}}^{2}\right) \tan \alpha \cos \alpha_{c} \sin \alpha_{c}-\left(m_{h_{1}}^{2}-m_{h_{2}}^{2} \sin ^{2} \alpha_{c}-m_{h_{3}}^{2} \cos ^{2} \alpha_{c}\right) \sin \alpha_{b}} .
$$

\section{B Tri-linear Higgs couplings}

From the quartic terms in the scalar potential eq. (2.1), we can obtain the interactions between three neutral scalars, in the basis of $\left(H_{1}^{0}, H_{2}^{0}, A^{0}\right)$,

$$
\begin{aligned}
\mathcal{L}_{3 s}= & \frac{1}{4}\left(A^{0}\right)^{3} \cos \beta\left\{2 \sin \beta \operatorname{Im} \lambda_{5}-\cos \beta \operatorname{Im} \lambda_{7}\right\} \\
+ & \frac{1}{8}\left(A^{0}\right)^{2}\left\{\left[-5 H_{1}^{0} \cos \beta+H_{1}^{0} \cos (3 \beta)-H_{2}^{0}(5 \sin \beta+\sin (3 \beta))\right] \operatorname{Re} \lambda_{5}\right. \\
& \left.\quad+4\left[H_{1}^{0} \cos \beta \sin ^{2} \beta \lambda_{1}+H_{2}^{0} \cos ^{2} \beta \sin \beta \lambda_{2}+\left(H_{1}^{0} \cos ^{3} \beta+H_{2}^{0} \sin ^{3} \beta\right)\left(\lambda_{3}+\lambda_{4}\right)\right]\right\} \\
& +\frac{1}{4} A^{0}\left\{\left[4 H_{1}^{0} H_{2}^{0}+\left(\left(H_{1}^{0}\right)^{2}+\left(H_{2}^{0}\right)^{2}\right) \sin (2 \beta)\right] \operatorname{Im} \lambda_{5}+H_{2}^{0}\left(2 H_{2}^{0}-H_{2}^{0} \cos (2 \beta)+H_{1}^{0} \sin (2 \beta)\right) \operatorname{Im} \lambda_{7}\right\} \\
& +\frac{1}{2}\left\{H_{2}^{0} \sin \beta\left[\left(H_{2}^{0}\right)^{2} \lambda_{2}+\left(H_{1}^{0}\right)^{2}\left(\lambda_{3}+\lambda_{4}+\operatorname{Re} \lambda_{5}\right)\right]+H_{1}^{0} \cos \beta\left[\left(H_{1}^{0}\right)^{2} \lambda_{1}+\left(H_{2}^{0}\right)^{2}\left(\lambda_{3}+\lambda_{4}+\operatorname{Re} \lambda_{5}\right)\right]\right\} .
\end{aligned}
$$


From these terms one can readily obtain the $h_{i} h_{j} h_{k}$ interactions in the mass eigenstate basis $\left(h_{1}, h_{2}, h_{3}\right)$ using the orthogonal matrix $R$ from eq. (2.4). In particular, the $g_{i 11}(i=2,3)$ coefficients used in eq. (2.24) are

$$
g_{i 11}=\frac{1}{2} \sum_{a \leq b \leq c} \frac{\partial^{3} \mathcal{L}_{3 s}}{\partial H_{a} \partial H_{b} \partial H_{c}} \frac{\partial H_{a}}{\partial h_{1}} \frac{\partial H_{b}}{\partial h_{1}} \frac{\partial H_{c}}{\partial h_{i}}=\frac{1}{2} \sum_{a \leq b \leq c} \frac{\partial^{3} \mathcal{L}_{3 s}}{\partial H_{a} \partial H_{b} \partial H_{c}} R_{1 a} R_{1 b} R_{i c},
$$

where $\left\{H_{a}\right\}=\left(H_{1}^{0}, H_{2}^{0}, A^{0}\right)$.

\section{Formula for $g-2$}

The magnetic and electric dipole moments of a fermion $f$ correspond to the real and imaginary parts of the Wilson coefficient $c$ of the effective operator

$$
\mathcal{L}_{\text {eff }}=c \bar{f}_{L} \sigma_{\mu \nu} f_{R} F^{\mu \nu}+\text { h.c. },
$$

where in the Type-I and Type-II 2HDMs we consider the main contributions to the coefficient $c$ that arise from the two-loop Barr-Zee type diagrams. It is straightforward to translate the electron EDM results to the corresponding muon anomalous dipole moment. The prescription for the translation is,

$a_{\mu}=\frac{2 m_{\mu}^{2}}{e Q_{\mu} m_{e}} \times \begin{cases}d_{e}^{\gamma}\left(\begin{array}{c}c_{e} \rightarrow \tilde{c}_{\mu} \\ \tilde{c}_{e} \rightarrow-c_{\mu}\end{array}\right), & h \gamma \gamma, h Z \gamma \text { diagrams } \\ d_{e}^{\gamma}\left(\operatorname{Im}\left(a_{W^{+} H^{-} h_{i}}\right) \rightarrow-\operatorname{Re}\left(a_{W^{+} H^{-} h_{i}}\right)\right), & W^{ \pm} H_{\mp} \gamma \text { diagrams }(\mathrm{S}) \\ d_{e}^{\gamma}\left(\operatorname{Im}\left(c_{\bar{t}_{R} b_{L} H^{+}}^{*} c_{\bar{\nu} e_{R} H^{+}}\right) \rightarrow-\operatorname{Re}\left(c_{\bar{t}_{R} b_{L} H^{+}}^{*} c_{\bar{\nu} e_{R} H^{+}}\right)\right), & W^{ \pm} H_{\mp} \gamma \text { diagrams }(\mathrm{F})\end{cases}$

where $A B \gamma$ corresponds to those Barr-Zee diagrams with $h_{1}$ lines connected to the upper loop, and the $\mathrm{S} / \mathrm{F}$ in the bracket corresponds to heavy Higgs scalars/SM fermions running in the upper loop. The $h \gamma \gamma, h Z \gamma$ and $W^{ \pm} H_{\mp} \gamma$ diagram (S) contributions to the EDM have been summarized in refs. [10, 71]. The $W^{ \pm} H_{\mp} \gamma$ diagram (F) contributions to the EDM vanish in $2 \mathrm{HDM}$ s with approximate $Z_{2}$ symmetry, but have been calculated in a more general framework in ref. [72]. We perform the above translation based on results in ref. [72]. See also ref. [73] for a recent work on $g-2$ in a 2 HDM.

We list below the analytic results for the contributions to the muon $g-2$ in a $2 \mathrm{HDM}$ :

$$
\begin{aligned}
&\left(\Delta a_{\mu}\right)_{f}^{h \gamma \gamma}= \frac{G_{F} m_{\mu}^{2} N_{c} Q_{f}^{2} \alpha}{2 \sqrt{2} \pi^{3}} \sum_{i=1}^{3}\left[-c_{f, i} c_{\mu, i} f\left(z_{f}^{i}\right)+\tilde{c}_{f, i} \tilde{c}_{\mu, i} g\left(z_{f}^{i}\right)\right], \\
&\left(\Delta a_{\mu}\right)_{f}^{h Z \gamma}=\frac{G_{F} m_{\mu}^{2} N_{c} Q_{t} g_{Z \bar{e} e}^{V} g_{Z \bar{f} f}^{V} \sum_{i=1}^{3}}{8 \sqrt{2} \pi^{4} Q_{\mu}}\left[-c_{f, i} c_{\mu, i} \tilde{f}\left(z_{f}^{i}, \frac{m_{f}^{2}}{M_{Z}^{2}}\right)+\tilde{c}_{f, i} \tilde{c}_{\mu, i} \tilde{g}\left(z_{f}^{i}, \frac{m_{f}^{2}}{M_{Z}^{2}}\right)\right], \\
&\left(\Delta a_{\mu}\right)_{W}^{h \gamma \gamma=} \frac{G_{F} m_{\mu}^{2} \alpha}{8 \sqrt{2} \pi^{3}} \sum_{i=1}^{3}\left[\left(6+\frac{1}{z_{w}^{i}}\right) f\left(z_{w}^{i}\right)+\left(10-\frac{1}{z_{w}^{i}}\right) g\left(z_{w}^{i}\right)\right]\left(-c_{\mu, i}\right) a_{i}, \\
&\left(\Delta a_{\mu}\right)_{W}^{h Z \gamma}=\frac{g_{Z \bar{f} f}^{V} g_{Z W W}}{Q_{\mu}} \frac{G_{F} m_{\mu}^{2}}{32 \sqrt{2} \pi^{4}} \sum_{i=1}^{3}\left[\left(6-\sec ^{2} \theta_{W}+\frac{2-\sec ^{2} \theta_{W}}{2 z_{w}^{i}}\right) \tilde{f}\left(z_{w}^{i}, \cos ^{2} \theta_{W}\right)\right. \\
&\left.+\left(10-3 \sec ^{2} \theta_{W}-\frac{2-\sec ^{2} \theta_{W}}{2 z_{w}^{i}}\right) \tilde{g}\left(z_{w}^{i}, \cos ^{2} \theta_{W}\right)\right]\left(-c_{\mu, i}\right) a_{i},
\end{aligned}
$$




$$
\begin{aligned}
\left(\Delta a_{\mu}\right)_{H^{+}}^{h \gamma \gamma} & =\frac{G_{F} m_{\mu}^{2} \alpha}{8 \sqrt{2} \pi^{3}}\left(\frac{v}{m_{H^{+}}}\right)^{2} \sum_{i=1}^{3}\left[f\left(z_{H}^{i}\right)-g\left(z_{H}^{i}\right)\right]\left(-c_{\mu, i}\right) \bar{\lambda}_{i} \\
\left(\Delta a_{\mu}\right)_{H^{+}}^{h Z \gamma} & =\frac{g_{Z \bar{f} f}^{V} g_{Z H^{+} H^{-}}}{Q_{\mu}} \frac{G_{F} m_{\mu}^{2}}{32 \sqrt{2} \pi^{4}}\left(\frac{v}{m_{H^{+}}}\right)^{2} \sum_{i=1}^{3}\left[\tilde{f}\left(z_{H}^{i}, m_{H^{+}}^{2} / M_{Z}^{2}\right)-\tilde{g}\left(z_{H}^{i}, m_{H^{+}}^{2} / M_{Z}^{2}\right)\right]\left(-c_{\mu, i}\right) \bar{\lambda}_{i}, \\
\left(\Delta a_{\mu}\right)_{H}^{H W \gamma} & =-\frac{G_{F} m_{\mu}^{2} c_{H^{+}} \bar{\nu} e^{-}}{64 \sqrt{2} \pi^{4} Q_{\mu}} \sum_{i}\left[\frac{e^{2}}{2 \sin ^{2} \theta_{W}} \mathcal{I}_{4}\left(m_{h_{i}}^{2}, m_{H^{+}}^{2}\right) a_{i}-\mathcal{I}_{5}\left(m_{h_{i}}^{2}, m_{H^{+}}^{2}\right) \bar{\lambda}_{i}\right]\left(-\operatorname{Re}\left(a_{W^{+} H^{-} h_{i}}\right)\right), \\
\left(\Delta a_{\mu}\right)_{t, b}^{H W \gamma} & =\left(\frac{3 g^{2}}{16 \pi^{2}}\right)\left(\frac{g^{2} m_{\mu}^{2}}{32 \pi^{2} M_{W}^{2}}\right)\left(-\operatorname{Re}\left(c_{\bar{t}_{R} b_{L} H^{+}}^{*} c_{H^{+} \nu e^{-}}\right)\right)\left(\frac{2}{3} F_{t}-\frac{1}{3} F_{b}\right),
\end{aligned}
$$

where $z_{f}^{i}=m_{f}^{2} / m_{h_{i}}^{2}(f=t, b), z_{w}^{i}=M_{W}^{2} / m_{h_{i}}^{2}, z_{H}^{i}=m_{H^{+}}^{2} / m_{h_{i}}^{2}$, and $c_{e, i}=c_{\mu, i}=c_{\tau, i}$, $\tilde{c}_{e, i}=\tilde{c}_{\mu, i}=\tilde{c}_{\tau, i}$ can be obtained from table 1 .

The relevant coefficients are,

$$
\begin{aligned}
g_{Z f \bar{f}}^{V}= & \frac{g}{2 \cos \theta_{W}}\left(T_{3}^{f}-2 Q^{f} \sin ^{2} \theta_{W}\right), \\
g_{W W Z}= & e \cot \theta_{W}, \\
g_{Z H^{+} H^{-}}= & \frac{1}{2} e \cot \theta_{W}\left(1-\tan ^{2} \theta_{W}\right), \\
\bar{\lambda}_{i}= & R_{i 1} \cdot\left(\lambda_{3} \cos ^{2} \beta+\left(\lambda_{1}-\lambda_{4}-\operatorname{Re} \lambda_{5}\right) \sin ^{2} \beta\right) \cos \beta \\
& +R_{i 2} \cdot\left(\lambda_{3} \sin ^{2} \beta+\left(\lambda_{2}-\lambda_{4}-\operatorname{Re} \lambda_{5}\right) \cos ^{2} \beta\right) \sin \beta \\
& +R_{i 3} \cdot \operatorname{Im} \lambda_{5} \sin \beta \cos \beta, \\
a_{W^{+} H^{-} h_{i}}= & -\sin \beta R_{i 1}+\cos \beta R_{i 2}+i R_{i 3}, \\
c_{\bar{t}_{R} b_{L} H^{+}}= & \cot \beta, \\
c_{H^{+} \bar{\nu} e^{-}}= & \begin{cases}\cot \beta & \text { Type I } \\
-\tan \beta & \text { Type II }\end{cases}
\end{aligned}
$$

The relevant loop functions are,

$$
\begin{aligned}
h_{0}(z) & =\frac{z^{4}}{2} \int_{0}^{1} d x \int_{0}^{1} d y \frac{x^{3} y^{3}(1-x)}{\left(z^{2} x(1-x y)+(1-y)(1-x)\right)^{2}}, \\
f(z) & =\frac{z}{2} \int_{0}^{1} d x \frac{1-2 x(1-x)}{x(1-x)-z} \log \frac{x(1-x)}{z}, \\
g(z) & =\frac{z}{2} \int_{0}^{1} d x \frac{1}{x(1-x)-z} \log \frac{x(1-x)}{z}, \\
h(z) & =\frac{z}{2} \int_{0}^{1} d x \frac{1}{z-x(1-x)}\left(1+\frac{z}{z-x(1-x)} \log \frac{x(1-x)}{z}\right), \\
\tilde{f}(x, y) & =\frac{y f(x)}{y-x}+\frac{x f(y)}{x-y}, \\
\tilde{g}(x, y) & =\frac{y g(x)}{y-x}+\frac{x g(y)}{x-y}, \\
\mathcal{I}_{4,5}\left(m_{1}^{2}, m_{2}^{2}\right) & =\frac{M_{W}^{2}}{m_{H^{+}}^{2}-M_{W}^{2}}\left(I_{4,5}\left(M_{W}^{2}, m_{1}^{2}\right)-I_{4,5}\left(m_{2}^{2}, m_{1}^{2}\right)\right)
\end{aligned}
$$




$$
\begin{aligned}
I_{4}\left(m_{1}^{2}, m_{2}^{2}\right)= & \int_{0}^{1} d z(1-z)^{2}\left(z-4+z \frac{m_{H^{+}}^{2}-m_{2}^{2}}{M_{W}^{2}}\right) \\
& \times \frac{m_{1}^{2}}{M_{W}^{2}(1-z)+m_{2}^{2} z-m_{1}^{2} z(1-z)} \log \frac{M_{W}^{2}(1-z)+m_{2}^{2} z}{m_{1}^{2} z(1-z)}, \\
I_{5}\left(m_{1}^{2}, m_{2}^{2}\right)= & \int_{0}^{1} d z \frac{m_{1}^{2} z(1-z)^{2}}{M_{H^{+}}^{2}(1-z)+m_{2}^{2} z-m_{1}^{2} z(1-z)} \log \frac{M_{H^{+}}^{2}(1-z)+m_{2}^{2} z}{m_{1}^{2} z(1-z)}, \\
\operatorname{Sp}(z)= & -\int_{0}^{z} t^{-1} \ln (1-t) d t, \\
T(z)= & \frac{1-3 z}{z^{2}} \frac{\pi^{2}}{6}-\left(\frac{1}{z}-\frac{5}{2}\right) \ln z-\frac{1}{z}-\left(2-\frac{1}{z}\right)\left(1-\frac{1}{z}\right) \operatorname{Sp}(1-z), \\
B(z)= & \frac{1}{z}+\frac{2 z-1}{z^{2}} \frac{\pi^{2}}{6}+\left(\frac{3}{2}-\frac{1}{z}\right) \ln z-\left(2-\frac{1}{z}\right) \frac{1}{z} \operatorname{Sp}(1-z), \\
F_{t}= & \frac{T\left(m_{H^{+}}^{2} / m_{t}^{2}\right)-T\left(M_{W}^{2} / m_{t}^{2}\right)}{m_{H^{+}}^{2} / m_{t}^{2}-M_{W}^{2} / m_{t}^{2}}, \\
F_{b}= & \frac{B\left(m_{H^{+}}^{2} / m_{t}^{2}\right)-B\left(M_{W}^{2} / m_{t}^{2}\right)}{m_{H^{+}}^{2} / m_{t}^{2}-M_{W}^{2} / m_{t}^{2}} .
\end{aligned}
$$

\section{Acknowledgments}

We thank Jing Shu and Michael Spira for useful discussions. The work of C.-Y. Chen and S. Dawson is supported by the U.S. Department of Energy under grant No. DE-AC0298CH10886 and contract DE-AC02-76SF00515. This work of Y. Zhang is supported by the Gordon and Betty Moore Foundation through Grant \#776 to the Caltech Moore Center for Theoretical Cosmology and Physics, and by the DOE Grant DE-FG02-92ER40701, and also by a DOE Early Career Award under Grant No. DE-SC0010255.

Open Access. This article is distributed under the terms of the Creative Commons Attribution License (CC-BY 4.0), which permits any use, distribution and reproduction in any medium, provided the original author(s) and source are credited.

\section{References}

[1] ATLAS collaboration, Observation of a new particle in the search for the standard model Higgs boson with the ATLAS detector at the LHC, Phys. Lett. B 716 (2012) 1 [arXiv: 1207.7214] [INSPIRE].

[2] CMS collaboration, Observation of a new boson at a mass of $125 \mathrm{GeV}$ with the CMS experiment at the LHC, Phys. Lett. B 716 (2012) 30 [arXiv:1207.7235] [INSPIRE].

[3] G.C. Branco et al., Theory and phenomenology of two-Higgs-doublet models, Phys. Rept. 516 (2012) 1 [arXiv:1106.0034] [INSPIRE].

[4] ATLAS collaboration, Constraints on new phenomena via Higgs coupling measurements with the ATLAS detector, ATLAS-CONF-2014-010, ATLAS-COM-CONF-2014-011 [INSPIRE].

[5] C.-Y. Chen and S. Dawson, Exploring two Higgs doublet models through Higgs production, Phys. Rev. D 87 (2013) 055016 [arXiv:1301.0309] [INSPIRE]. 
[6] B. Coleppa, F. Kling and S. Su, Constraining type II 2HDM in light of LHC Higgs searches, JHEP 01 (2014) 161 [arXiv: 1305.0002] [INSPIRE].

[7] K. Cheung, J.S. Lee and P.-Y. Tseng, Higgcision in the two-Higgs doublet models, JHEP 01 (2014) 085 [arXiv: 1310.3937] [INSPIRE].

[8] J. Shu and Y. Zhang, Impact of a CP-violating Higgs sector: from LHC to baryogenesis, Phys. Rev. Lett. 111 (2013) 091801 [arXiv: 1304.0773] [INSPIRE].

[9] D.E. Morrissey and M.J. Ramsey-Musolf, Electroweak baryogenesis, New J. Phys. 14 (2012) 125003 [arXiv:1206.2942] [InSPIRE].

[10] S. Inoue, M.J. Ramsey-Musolf and Y. Zhang, CP-violating phenomenology of flavor conserving two Higgs doublet models, Phys. Rev. D 89 (2014) 115023 [arXiv:1403.4257] [INSPIRE].

[11] J. Brod, U. Haisch and J. Zupan, Constraints on CP-violating Higgs couplings to the third generation, JHEP 11 (2013) 180 [arXiv:1310.1385] [INSPIRE].

[12] D. Fontes, J.C. Romão, R. Santos and J.P. Silva, Large pseudoscalar Yukawa couplings in the complex 2HDM, arXiv: 1502.01720 [INSPIRE].

[13] L. Lavoura and J.P. Silva, Fundamental CP-violating quantities in a $\mathrm{SU}(2) \times \mathrm{U}(1)$ model with many Higgs doublets, Phys. Rev. D 50 (1994) 4619 [hep-ph/9404276] [INSPIRE].

[14] A. Barroso, P.M. Ferreira, R. Santos and J.P. Silva, Probing the scalar-pseudoscalar mixing in the $125 \mathrm{GeV}$ Higgs particle with current data, Phys. Rev. D 86 (2012) 015022 [arXiv: 1205.4247] [INSPIRE].

[15] CMS collaboration, Constraints on the spin-parity and anomalous HVV couplings of the Higgs boson in proton collisions at 7 and $8 \mathrm{TeV}$, arXiv:1411.3441 [INSPIRE].

[16] ATLAS collaboration, Measurements of the properties of the Higgs-like boson in the four lepton decay channel with the ATLAS detector using $25 \mathrm{fb}^{-1}$ of proton-proton collision data, ATLAS-CONF-2013-013 [INSPIRE].

[17] R. Harnik, A. Martin, T. Okui, R. Primulando and F. Yu, Measuring CP-violation in $h \rightarrow \tau^{+} \tau^{-}$at colliders, Phys. Rev. D 88 (2013) 076009 [arXiv:1308.1094] [InSPIRE].

[18] F. Bishara et al., Probing CP-violation in $h \rightarrow \gamma \gamma$ with converted photons, JHEP 04 (2014) 084 [arXiv: 1312.2955] [INSPIRE].

[19] Y. Chen, A. Falkowski, I. Low and R. Vega-Morales, New observables for CP-violation in Higgs decays, Phys. Rev. D 90 (2014) 113006 [arXiv:1405.6723] [InSPIRE].

[20] M.J. Dolan, P. Harris, M. Jankowiak and M. Spannowsky, Constraining CP-violating Higgs sectors at the LHC using gluon fusion, Phys. Rev. D 90 (2014) 073008 [arXiv:1406.3322] [INSPIRE].

[21] S. Berge, W. Bernreuther and S. Kirchner, Determination of the Higgs CP mixing angle in the tau decay channels at the LHC including the Drell-Yan background, Eur. Phys. J. C 74 (2014) 3164 [arXiv:1408.0798] [InSPIRE].

[22] X.-G. He, G.-N. Li and Y.-J. Zheng, Probing Higgs boson CP properties with $t \bar{t} H$ at the LHC and the $100 \mathrm{TeV}$ pp collider, arXiv:1501.00012 [INSPIRE].

[23] S. Bar-Shalom, D. Atwood, G. Eilam, R.R. Mendel and A. Soni, Large tree level CP-violation in $e^{+} e^{-} \rightarrow t \bar{t} H^{0}$ in the two-Higgs-doublet model, Phys. Rev. D 53 (1996) 1162 [hep-ph/9508314] [INSPIRE]. 
[24] D. Atwood, S. Bar-Shalom, G. Eilam and A. Soni, CP violation in top physics, Phys. Rept. 347 (2001) 1 [hep-ph/0006032] [INSPIRE].

[25] S. Ipek, Perturbative analysis of the electron electric dipole moment and CP-violation in two-Higgs-doublet models, Phys. Rev. D 89 (2014) 073012 [arXiv:1310.6790] [INSPIRE].

[26] K. Cheung, J.S. Lee, E. Senaha and P.-Y. Tseng, Confronting Higgcision with electric dipole moments, JHEP 06 (2014) 149 [arXiv: 1403.4775] [inSPIRE].

[27] L. Bian, T. Liu and J. Shu, Post-ACME2013 CP-violation in Higgs physics and electroweak baryogenesis, arXiv:1411.6695 [INSPIRE].

[28] M. Carena, J.R. Ellis, S. Mrenna, A. Pilaftsis and C.E.M. Wagner, Collider probes of the MSSM Higgs sector with explicit CP-violation, Nucl. Phys. B 659 (2003) 145 [hep-ph/0211467] [INSPIRE].

[29] P. Bechtle et al., HiggsBounds-4: improved tests of extended Higgs sectors against exclusion bounds from LEP, the Tevatron and the LHC, Eur. Phys. J. C 74 (2014) 2693 [arXiv: 1311.0055] [INSPIRE].

[30] A. Arbey, J. Ellis, R.M. Godbole and F. Mahmoudi, Exploring CP-violation in the MSSM, Eur. Phys. J. C 75 (2015) 85 [arXiv:1410.4824] [INSPIRE].

[31] B. Li and C.E.M. Wagner, CP-odd component of the lightest neutral Higgs boson in the $M S S M$, arXiv: 1502.02210 [INSPIRE].

[32] A. Chakraborty et al., $125 \mathrm{GeV}$ Higgs signal at the LHC in the CP-violating MSSM, Phys. Rev. D 90 (2014) 055005 [arXiv: 1301.2745] [INSPIRE].

[33] M. Carena, H.E. Haber, I. Low, N.R. Shah and C.E.M. Wagner, Complementarity between nonstandard Higgs boson searches and precision Higgs boson measurements in the MSSM, Phys. Rev. D 91 (2015) 035003 [arXiv: 1410.4969] [InSPIRE].

[34] C.-Y. Chen, S. Dawson and M. Sher, Heavy Higgs searches and constraints on two Higgs doublet models, Phys. Rev. D 88 (2013) 015018 [arXiv:1305.1624] [INSPIRE].

[35] N. Craig, J. Galloway and S. Thomas, Searching for signs of the second Higgs doublet, arXiv:1305.2424 [INSPIRE].

[36] A. Freitas and P. Schwaller, Higgs CP properties from early LHC data, Phys. Rev. D 87 (2013) 055014 [arXiv:1211.1980] [inSPIRE].

[37] A. Djouadi and G. Moreau, The couplings of the Higgs boson and its CP properties from fits of the signal strengths and their ratios at the 7+8 TeV LHC, Eur. Phys. J. C 73 (2013) 2512 [arXiv: 1303.6591] [INSPIRE].

[38] W.-F. Chang, W.-P. Pan and F. Xu, Effective gauge-Higgs operators analysis of new physics associated with the Higgs boson, Phys. Rev. D 88 (2013) 033004 [arXiv:1303.7035] [INSPIRE].

[39] A.J. Buras, G. Isidori and P. Paradisi, EDMs versus $C P V$ in $B_{s, d}$ mixing in two Higgs doublet models with MFV, Phys. Lett. B 694 (2011) 402 [arXiv:1007.5291] [INSPIRE].

[40] J.M. Cline, K. Kainulainen and M. Trott, Electroweak baryogenesis in two Higgs doublet models and B meson anomalies, JHEP 11 (2011) 089 [arXiv:1107.3559] [INSPIRE].

[41] M. Jung and A. Pich, Electric dipole moments in two-Higgs-doublet models, JHEP 04 (2014) 076 [arXiv: 1308.6283] [InSPIRE]. 
[42] LHC Higgs Cross Section Working Group collaboration, S. Dittmaier et al., Handbook of LHC Higgs cross sections: 1. Inclusive observables, arXiv:1101.0593 [INSPIRE].

[43] https://twiki.cern.ch/twiki/bin/view/LHCPhysics/CrossSections.

[44] LHC Higgs Cross Section Working Group collaboration, S. Heinemeyer et al., Handbook of LHC Higgs cross sections: 3. Higgs properties, arXiv:1307.1347 [INSPIRE].

[45] http://tiger.web.psi.ch/grids/.

[46] F. Maltoni, G. Ridolfi and M. Ubiali, b-initiated processes at the LHC: a reappraisal, JHEP 07 (2012) 022 [Erratum ibid. 04 (2013) 095] [arXiv: 1203.6393] [INSPIRE].

[47] ATLAS collaboration, Search for Higgs bosons in two-Higgs-doublet models in the $H \rightarrow W W \rightarrow e \nu \mu \nu$ channel with the ATLAS detector, ATLAS-CONF-2013-027, ATLAS-COM-CONF-2013-005 [INSPIRE].

[48] CMS collaboration, Search for a standard-model-like Higgs boson with a mass in the range 145 to $1000 \mathrm{GeV}$ at the LHC, Eur. Phys. J. C 73 (2013) 2469 [arXiv:1304.0213] [CMS-HIG-12-034] [INSPIRE].

[49] ATLAS collaboration, Search for a high-mass Higgs boson in the $H \rightarrow W W \rightarrow l \nu l \nu$ decay channel with the ATLAS detector using $21 \mathrm{fb}^{-1}$ of proton-proton collision data, ATLAS-CONF-2013-067, ATLAS-COM-CONF-2013-082 [INSPIRE].

[50] CMS collaboration, Search for a pseudoscalar boson $A$ decaying into a $Z$ and an $h$ boson in the $\ell \ell b \bar{b}$ final state, CMS-PAS-HIG-14-011 [INSPIRE].

[51] ATLAS collaboration, Search for a CP-odd Higgs boson decaying to Zh in pp collisions at $\sqrt{s}=8 \mathrm{TeV}$ with the ATLAS detector, Phys. Lett. B 744 (2015) 163 [arXiv:1502.04478] [INSPIRE].

[52] CMS collaboration, Search for neutral MSSM Higgs bosons decaying to a pair of tau leptons in pp collisions, JHEP 10 (2014) 160 [arXiv:1408.3316] [CMS-HIG-13-021] [INSPIRE].

[53] A. Djouadi and J. Quevillon, The MSSM Higgs sector at a high $M_{\mathrm{SUSY}}$ : reopening the low $\tan \beta$ regime and heavy Higgs searches, JHEP 10 (2013) 028 [arXiv:1304.1787] [INSPIRE].

[54] CMS collaboration, Precise determination of the mass of the Higgs boson and tests of compatibility of its couplings with the standard model predictions using proton collisions at 7 and $8 \mathrm{TeV}$, arXiv: 1412.8662 [CMS-HIG-14-009] [INSPIRE].

[55] ATLAS collaboration, Observation and measurement of Higgs boson decays to $W W^{*}$ with the ATLAS detector, arXiv:1412.2641 [INSPIRE].

[56] ATLAS collaboration, Updated coupling measurements of the Higgs boson with the ATLAS detector using up to $25 \mathrm{fb}^{-1}$ of proton-proton collision data, ATLAS-CONF-2014-009 [INSPIRE].

[57] ATLAS collaboration, Measurement of Higgs boson production in the diphoton decay channel in pp collisions at center-of-mass energies of 7 and $8 \mathrm{TeV}$ with the ATLAS detector, Phys. Rev. D 90 (2014) 112015 [arXiv: 1408.7084] [INSPIRE].

[58] ATLAS collaboration, Search for the bb decay of the standard model Higgs boson in associated W/ZH production with the ATLAS detector, ATLAS-CONF-2013-079 [INSPIRE].

[59] ATLAS collaboration, Evidence for the Higgs-boson Yukawa coupling to tau leptons with the ATLAS detector, JHEP 04 (2015) 117 [arXiv:1501.04943] [INSPIRE]. 
[60] J. Engel, M.J. Ramsey-Musolf and U. van Kolck, Electric dipole moments of nucleons, nuclei and atoms: the standard model and beyond, Prog. Part. Nucl. Phys. 71 (2013) 21 [arXiv: 1303.2371] [INSPIRE].

[61] C.-Y. Chen, Projections for two Higgs doublet models at the LHC and ILC: a Snowmass white paper, arXiv:1308.3487 [INSPIRE].

[62] P.M. Ferreira, J.F. Gunion, H.E. Haber and R. Santos, Probing wrong-sign Yukawa couplings at the LHC and a future linear collider, Phys. Rev. D 89 (2014) 115003 [arXiv:1403.4736] [INSPIRE].

[63] F. Mahmoudi and O. Stål, Flavor constraints on the two-Higgs-doublet model with general Yukawa couplings, Phys. Rev. D 81 (2010) 035016 [arXiv:0907.1791] [INSPIRE].

[64] A. Wahab El Kaffas, P. Osland and O.M. Ogreid, Constraining the two-Higgs-doublet-model parameter space, Phys. Rev. D 76 (2007) 095001 [arXiv:0706.2997] [INSPIRE].

[65] W. Grimus, L. Lavoura, O.M. Ogreid and P. Osland, The oblique parameters in multi-Higgs-doublet models, Nucl. Phys. B 801 (2008) 81 [arXiv:0802.4353] [inSPIRE].

[66] H.-J. He, N. Polonsky and S.-f. Su, Extra families, Higgs spectrum and oblique corrections, Phys. Rev. D 64 (2001) 053004 [hep-ph/0102144] [INSPIRE].

[67] H.E. Haber and D. O'Neil, Basis-independent methods for the two-Higgs-doublet model. III. The CP-conserving limit, custodial symmetry and the oblique parameters $S, T, U$, Phys. Rev. D 83 (2011) 055017 [arXiv: 1011.6188] [INSPIRE].

[68] R. Barbieri, L.J. Hall and V.S. Rychkov, Improved naturalness with a heavy Higgs: an alternative road to LHC physics, Phys. Rev. D 74 (2006) 015007 [hep-ph/0603188] [INSPIRE].

[69] GFitter Group collaboration, M. Baak et al., The global electroweak fit at NNLO and prospects for the LHC and ILC, Eur. Phys. J. C 74 (2014) 3046 [arXiv:1407.3792] [INSPIRE].

[70] M. Davier and W.J. Marciano, The theoretical prediction for the muon anomalous magnetic moment, Ann. Rev. Nucl. Part. Sci. 54 (2004) 115 [inSPIRE].

[71] T. Abe, J. Hisano, T. Kitahara and K. Tobioka, Gauge invariant Barr-Zee type contributions to fermionic EDMs in the two-Higgs doublet models, JHEP 01 (2014) 106 [arXiv: 1311.4704] [INSPIRE].

[72] D. Bowser-Chao, D. Chang and W.-Y. Keung, Electron electric dipole moment from CP-violation in the charged Higgs sector, Phys. Rev. Lett. 79 (1997) 1988 [hep-ph/9703435] [INSPIRE].

[73] V. Ilisie, New Barr-Zee contributions to $(g-2)_{\mu}$ in two-Higgs-doublet models, arXiv:1502.04199 [INSPIRE]. 